\title{
Open-string T-duality and applications to non-geometric backgrounds
}

\author{
Fabrizio Cordonier-Tello, ${ }^{a}$ Dieter Lüst ${ }^{a, b, c}$ and Erik Plauschinn ${ }^{a}$ \\ ${ }^{a}$ Arnold Sommerfeld Center for Theoretical Physics, Ludwig-Maximilians-Universität, \\ Theresienstraße 37, 80333 München, Germany \\ ${ }^{b}$ Max-Planck-Institut für Physik, \\ Föhringer Ring 6, 80805 München, Germany \\ ${ }^{c}$ CERN, Theory Department, \\ 1211 Geneva 23, Switzerland \\ E-mail: f.cordonier@physik.uni-muenchen.de, dieter.luest@lmu.de, \\ erik.plauschinn@lmu.de
}

ABSTRACT: We revisit T-duality transformations for the open string via Buscher's procedure and work-out technical details which have been missing so far in the literature. We take into account non-trivial topologies of the world-sheet, we consider T-duality along directions with Neumann as well as Dirichlet boundary conditions, and we include collective T-duality along multiple directions.

We illustrate this formalism with the example of the three-torus with $H$-flux and its T-dual backgrounds, and we discuss global properties of open-string boundary conditions on such spaces.

Keywords: String Duality, D-branes

ARXiv EPRINT: 1806.01308 


\section{Contents}

1 Introduction 1

2 Non-linear sigma-model for the open string 4

2.1 World-sheet action 4

$\begin{array}{ll}2.2 & \text { Gauged world-sheet action }\end{array}$

$\begin{array}{lll}2.3 & \text { Recovering the ungauged world-sheet action } & 9\end{array}$

3 T-duality 10

3.1 Closed-string sector 11

$\begin{array}{lll}3.2 & \text { Open-string sector — Neumann directions } & 15\end{array}$

$\begin{array}{lll}3.3 & \text { Open-string sector — Dirichlet directions } & 18\end{array}$

4 Examples - three-torus with $H$-flux $\quad 19$

4.1 One T-duality 20

4.2 Two T-dualities 25

$\begin{array}{lll}4.3 & \text { Three T-dualities } & 27\end{array}$

5 Freed-Witten anomaly and boundary conditions 28

5.1 Freed-Witten anomaly 28

$\begin{array}{lll}5.2 & \text { Boundary conditions } & 29\end{array}$

6 Summary and conclusion $\quad 32$

\section{Introduction}

Dualities are remarkable features of string theory which have helped to expose and understand some of the intricate structures of the theory. Examples for dualities are S-duality, T-duality, mirror symmetry and heterotic-type I duality - and in this work we will be interested in T-duality for open strings.

Non-geometric backgrounds. Dualities can be used to construct new backgrounds for string theory which are well-defined only using duality transformations. An example is Ftheory in which S-duality is needed to construct globally-defined solutions, and in a similar way T-duality can be utilized to build so-called non-geometric backgrounds. The latter are spaces which do not allow for a description in terms of Riemannian geometry, but in which $O(D, D ; \mathbb{Z})$ T-duality transformations are used as transition functions between local charts $[1,2]$. In this way globally well-defined backgrounds can be constructed.

The standard example for a non-geometric background is obtained by applying successive or collective T-duality transformations to a three-torus with $H$-flux. After one 
T-duality one arrives at a twisted three-torus [3, 4], which is a geometric space and to which one can associate a geometric flux $F^{k}{ }_{i j}$. A second T-duality transformation gives the T-fold background [2], which locally can be expressed in terms of a metric and $B$-field but which globally is non-geometric. To this configuration one can associate a $Q$-flux $Q_{i}{ }^{j k}$. Finally, even though the Buscher rules cannot be applied, a formal third T-duality leads to a background with $R$-flux $R^{i j k}[5,6]$ and it has been argued that this space not even locally allows for a geometric description. This chain of T-duality transformations is often summarized as follows

$$
H_{i j k} \quad \stackrel{T_{i}}{\longrightarrow} \quad F_{j k}^{i} \quad \stackrel{T_{j}}{\longrightarrow} \quad Q_{k}{ }^{i j} \stackrel{T_{k}}{\longrightarrow} R^{i j k},
$$

where $T_{i}$ denotes a T-duality transformation along the direction labelled by $i$. Nongeometric backgrounds have interesting properties, for instance, they lead to noncommutative [7-13] and non-associative [14-22] structures (for a review see [23]). Furthermore, non-geometric $Q$ - and $R$-fluxes can help in stabilizing moduli in stringphenomenology (see e.g. [5, 6, 24-29]) and they can provide mechanisms for the construction of potentials for inflationary scenarios in string-cosmology (see e.g. [30, 31]).

The purpose of this paper is to investigate non-geometric backgrounds from an openstring perspective. In particular, we want to understand the global properties of D-branes in non-geometric spaces, which is important for applications in D-brane model building. In the context of doubled geometry, D-branes in non-geometric backgrounds have been discussed already in the original paper [2] and have been investigated further in [32, 33]. However, here we are interested in a description in string theory.

T-duality for open strings. In order to analyze D-branes in non-geometric backgrounds, we follow a strategy similar to the closed-string situation and apply T-duality transformations to D-brane configurations on a three-torus with $H$-flux.

T-duality for open strings in toroidal backgrounds with constant metric and $B$-field can be studied via well-known conformal-field theory (CFT) techniques, but for curved backgrounds with non-trivial $B$-field one usually has to rely on Buscher's procedure [34] of gauging a symmetry of the world-sheet action and integrating-out the gauge field. This approach has been investigated already in the literature, and some of the relevant papers are the following:

- In [35] the authors discuss T-duality for the open string along a single direction. They consider the bosonic string as well as the superstring from a world-sheet point of view, and they analyze the behavior of the effective target-space action. The direction along which the T-duality transformation is performed has Neumann boundary conditions, and a Lagrange multiplier is implemented in a way which does not allow for a generalization to Dirichlet directions for non-trivial world-sheet topologies.

- In $[36,37]$ the case of a single T-duality transformation is studied from a pathintegral point of view and as a canonical transformation. The gauge group on a stack of D-branes can be non-abelian, and the $B$-field is required to be independent of the coordinate along which T-duality is performed. T-duality is considered along a 
direction with Neumann boundary condition, and the topology of the world-sheet is assumed to be trivial.

- In [38-40] the authors investigate T-duality along multiple directions with a nonabelian isometry group. The $B$-field and consequently the $H$-flux are set to zero, and the topology of the world-sheet is assumed to be trivial.

- T-duality for open-string sigma models has been studied also in [41] with the inclusion of the fermionic sector on the world-sheet. Boundary conditions are discussed and the case of a single T-duality is considered for world-sheets with trivial topology.

In the present paper we extend the above analyses and work-out missing details: 1) we include world-sheets with a non-trivial topology in our studies, 2) we present procedures for T-duality transformations along directions with Neumann as well as Dirichlet boundary conditions, and 3) we include the case of performing collective T-duality transformations along multiple directions. For part of our analysis we include the case of a non-abelian isometry algebra, but final results are obtained only for the abelian case.

We furthermore mention that T-duality for open strings has been studied via canonical transformations also in [42, 43], and T-duality for D-branes from an effective field theory point of view has been investigated for instance in [44-46]. D-branes in non-geometric backgrounds have been analyzed from an effective field theory point of view in [15] and through boundary states in [47], and in the context of generalized complex geometry Dbranes and T-duality have been studied in [48]. Poisson-Lie T-duality for open strings has been discussed in [49] and [50], and in a somewhat different approach T-duality for open strings has also been considered in [51-53].

Outline. This paper consists of essentially two parts: first we discuss on general grounds open-string T-duality via Buscher's procedure, and in the second part we apply this formalism to D-brane configurations on a three-torus with $H$-flux. More concretely:

- In section 2 we consider the world-sheet description of open strings, the gauging of symmetries and how the ungauged action can be obtained from the gauged one. We pay special attention to the topology of the world-sheet.

- In section 3 we perform collective T-duality transformations either along all Neumann or all Dirichlet directions. We find for instance that, as expected, under T-duality Neumann and Dirichlet boundary conditions are interchanged.

- In section 4 we consider D-branes on a three-torus with $H$-flux and study T-duality along one, two and three directions. This is on the one-hand to illustrate the procedure discussed in section 3 , and on the other-hand to obtain explicit examples for D-branes on the twisted three-torus and on the T-fold.

- In section 5 we discuss the results from section 4 . We review the Freed-Witten anomaly cancellation condition, and we show that D-branes satisfying this condition are globally well-defined on the twisted torus and on the T-fold.

- Section 6 contains a brief summary of the results obtained in this paper. 


\section{Non-linear sigma-model for the open string}

We begin our discussion by reviewing the non-linear sigma-model description of the open string and studying the gauging of world-sheet symmetries. The latter are then employed in section 3 for performing T-duality transformations.

\subsection{World-sheet action}

We first introduce the sigma-model for the open string, specify boundary conditions and consider global symmetries of the world-sheet action.

The action. The world-sheet action for an open string can be defined on a two-dimensional world-sheet $\Sigma$ with non-empty boundary $\partial \Sigma \neq \varnothing$. We allow for a non-trivial targetspace metric $G$, Kalb-Ramond field $B$, dilaton $\phi$ and open-string gauge field $a$, although at a later stage we impose restrictions upon them. The field strengths of $B$ and $a$ will be denoted by $H=\mathrm{d} B$ and $F=\mathrm{d} a$, respectively. For later convenience we perform a Wick rotation and consider an Euclidean metric on the world-sheet. The resulting action then reads

$$
\begin{aligned}
\mathcal{S}= & -\frac{1}{2 \pi \alpha^{\prime}} \int_{\Sigma}\left[\frac{1}{2} G_{i j} \mathrm{~d} X^{i} \wedge \star \mathrm{d} X^{j}+\frac{i}{2} B_{i j} \mathrm{~d} X^{i} \wedge \mathrm{d} X^{j}+\frac{\alpha^{\prime}}{2} \mathrm{R} \phi \star 1\right] \\
& -\frac{1}{2 \pi \alpha^{\prime}} \int_{\partial \Sigma}\left[2 \pi i \alpha^{\prime} a_{i} \mathrm{~d} X^{i}+\alpha^{\prime} k(s) \phi \mathrm{d} s\right]
\end{aligned}
$$

where $a=a_{i} \mathrm{~d} X^{i}$ is understood to be restricted to the boundary $\partial \Sigma$. Coordinates on the world-sheet $\Sigma$ are denoted as $\sigma^{a}=\left\{\sigma^{1}, \sigma^{2}\right\}$ and on the boundary $\partial \Sigma$ as $s$. Coordinates on a $D$-dimensional target-space are denoted by $X^{i}(\sigma)$ with $i=1, \ldots, D$, which can be interpreted as maps from the world-sheet $\Sigma$ to the target-space. The exterior derivative operator $\mathrm{d}$ acting on $X^{i}$ can therefore be written as $\mathrm{d} X^{i}=\partial_{\mathrm{a}} X^{i}(\sigma) \mathrm{d} \sigma^{\mathrm{a}}$. The Hodge star-operator on $\Sigma$ is denoted by $\star$, the Ricci scalar for the world-sheet metric is R, and $k(s)$ is the extrinsic curvature of the boundary $k=t^{\mathrm{a}} t^{\mathrm{b}} \nabla_{\mathrm{a}} n_{\mathrm{b}}$, where $t^{\mathrm{a}}$ and $n^{\mathrm{a}}$ are unit vectors tangential and normal to the boundary, respectively. Note furthermore that on the boundary we have $\left.\mathrm{d} X^{i}\right|_{\partial \Sigma}=t^{\mathrm{a}} \partial_{\mathrm{a}} X^{i} \mathrm{~d} s$.

Boundary conditions. Next, we consider the equations of motion for the fields $X^{i}$ and, in particular, the corresponding boundary conditions. Denoting by $\Gamma_{j k}^{i}$ the Christoffel symbols for the target-space metric $G_{i j}$ and by $H_{i j k}$ the components of the field strength $H=\mathrm{d} B$, the equations of motion for $X^{i}$ following from (2.1) read

$$
\begin{aligned}
0= & \mathrm{d} \star \mathrm{d} X^{i}+\Gamma_{m n}^{i} \mathrm{~d} X^{m} \wedge \star \mathrm{d} X^{n}-\frac{i}{2} H_{m n}^{i} \mathrm{~d} X^{m} \wedge \mathrm{d} X^{n} \\
& -\frac{\alpha^{\prime}}{2} G^{i m} \partial_{m} \phi \mathrm{R} \star 1,
\end{aligned}
$$

where the index of $H_{i j k}$ has been raised using the inverse of $G_{i j}$. As boundary condition we can impose Dirichlet boundary conditions of the form $\left.\delta X^{i}\right|_{\partial \Sigma}=0$ or Neumann boundary conditions. Denoting the tangential and normal part of $\mathrm{d} X^{i}$ on the boundary by

$$
\left.\left(\mathrm{d} X^{i}\right)_{\tan } \equiv t^{\mathrm{a}} \partial_{\mathrm{a}} X^{i} \mathrm{~d} s\right|_{\partial \Sigma},\left.\quad\left(\mathrm{d} X^{i}\right)_{\text {norm }} \equiv n^{\mathrm{a}} \partial_{\mathrm{a}} X^{i} \mathrm{~d} s\right|_{\partial \Sigma}
$$


where $t^{\mathrm{a}}$ and $n^{\mathrm{a}}$ are again unit tangential and normal vectors, and introducing the gaugeinvariant open-string field-strength $\mathcal{F}$ as $2 \pi \alpha^{\prime} \mathcal{F}=2 \pi \alpha^{\prime} F+B$, we can summarize the boundary conditions as

$$
\begin{array}{ll}
\text { Dirichlet } & 0=\left(\mathrm{d} X^{\hat{i}}\right)_{\tan }, \\
\text { Neumann } & 0=G_{a i}\left(\mathrm{~d} X^{i}\right)_{\text {norm }}+2 \pi \alpha^{\prime} i \mathcal{F}_{a b}\left(\mathrm{~d} X^{b}\right)_{\tan }+\left.\alpha^{\prime} k(s) \partial_{a} \phi \mathrm{d} s\right|_{\partial \Sigma} .
\end{array}
$$

Here and in the following we split the target-space index $i=1, \ldots, D$ into Dirichlet and Neumann directions $\hat{i}$ and $a$, respectively.

Now, the Hodge decomposition theorem for manifolds with boundary (see for instance [54]) allows us to decompose the space of closed $p$-forms $C^{p}=\left\{\omega \in \Omega^{p}: \mathrm{d} \omega=0\right\}$ into exact $p$-forms $E^{p}=\left\{\omega \in \Omega^{p}: \omega=\mathrm{d} \eta, \eta \in \Omega^{p-1}\right\}$ and closed and co-closed forms whose normal part vanishes on the boundary $C c C_{N}^{p}=\left\{\omega \in \Omega^{p}: \mathrm{d} \omega=0, \mathrm{~d}^{\dagger} \omega=0, \omega_{\text {norm }}=0\right\}$. Here, $\Omega^{p}$ is the space of smooth differential forms on $\Sigma$ and $\mathrm{d}^{\dagger}$ denotes the co-differential. In formulas, this decomposition reads

$$
C^{p}=E^{p} \oplus C c C_{N}^{p} .
$$

For Dirichlet boundary conditions - with vanishing tangential part of $\mathrm{d} X^{\hat{i}}$ - this implies in particular that the $C c C_{N}^{1}$ part of $\mathrm{d} X^{\hat{i}}$ vanishes and hence $\mathrm{d} X^{\hat{i}}$ is an exact one-form on the world-sheet $\Sigma$.

Global symmetries. In order to perform T-duality transformations, let us now require the world-sheet action (2.1) to be invariant under global symmetry transformations of the form

$$
\delta_{\epsilon} X^{i}=\epsilon^{\alpha} k_{\alpha}^{i} .
$$

For global symmetries the infinitesimal transformation parameters $\epsilon^{\alpha}$ are constant, and we require the target-space vectors $k_{\alpha}$ to satisfy a Lie algebra $\mathfrak{g}$ as

$$
\left[k_{\alpha}, k_{\beta}\right]_{L}=f_{\alpha \beta}{ }^{\gamma} k_{\gamma},
$$

where $f_{\alpha \beta} \gamma$ are the structure constants satisfying the Jacobi identity. The label $\alpha$ takes values $\alpha=1, \ldots, N$ with $N=\operatorname{dim}(\mathfrak{g})$.

The variation of the action (2.1) with respect to the transformations (2.6) does not vanish in general. However, when the following conditions are met then (2.6) is a global symmetry of the action

$$
\begin{aligned}
& \mathcal{L}_{k_{\alpha}} G=0, \\
& \mathcal{L}_{k_{\alpha}} B=\mathrm{d} v_{\alpha},\left.\quad 2 \pi \alpha^{\prime} \mathcal{L}_{k_{\alpha}} a\right|_{\partial \Sigma}=\left.\left(-v_{\alpha}+\mathrm{d} \omega_{\alpha}\right)\right|_{\partial \Sigma}, \\
& \mathcal{L}_{k_{\alpha}} \phi=0 \text {. }
\end{aligned}
$$

Here, $G=\frac{1}{2} G_{i j} \mathrm{~d} X^{i} \otimes \mathrm{d} X^{j}, B=\frac{1}{2} B_{i j} \mathrm{~d} X^{i} \wedge \mathrm{d} X^{j}, a=a_{i} \mathrm{~d} X^{i}$ and $\phi$ are interpreted as targetspace quantities. ${ }^{1}$ The Lie derivative along a vector field $k$ is given by $\mathcal{L}_{k}=\operatorname{do} \iota_{k}+\iota_{k}$ od, with

\footnotetext{
${ }^{1}$ In order to keep our notation manageable, we do not explicitly distinguish between quantities on the world-sheet and on target space, but assume this to be clear from the context. For instance, on the world-sheet we have $\mathcal{L}_{k} G=\frac{1}{2}\left(\mathcal{L}_{k} G\right)_{i j} d X^{i} \wedge \star d X^{j}$.
} 
$\iota$ the contraction operator acting on $\mathrm{d} X^{i}$ as $\iota_{\partial_{i}} \mathrm{~d} X^{j}=\delta_{i}{ }^{j}$ and $\mathrm{d}$ is the exterior derivative acting as $\mathrm{d} \equiv \mathrm{d} X^{i} \partial_{i}$. Furthermore, in order to apply Stokes' theorem and show that (2.6) is a global symmetry of the action (2.1) we require

$$
\begin{aligned}
& v_{\alpha} \ldots \text { globally-defined one-forms on } \Sigma, \\
& \omega_{\alpha} \ldots \text { globally-defined functions on } \partial \Sigma .
\end{aligned}
$$

Let us also investigate when the boundary conditions (2.4) are invariant under the global symmetry (2.6). For Neumann boundary conditions there are no restrictions due to (2.8), whereas for Dirichlet boundary conditions we find a non-trivial requirement. These are summarized as

$$
\begin{array}{ll}
\text { Dirichlet } & 0=\left.\partial_{a} k_{\alpha}^{\hat{i}}\right|_{\partial \Sigma}, \\
\text { Neumann } & \varnothing .
\end{array}
$$

However, strictly speaking the Dirichlet conditions read $\left.\delta X^{\hat{i}}\right|_{\partial \Sigma}=0$ which are not preserved under global transformations of the form (2.6). For local symmetry transformations on the other hand, the situation is different and we can preserve Dirichlet conditions of this form.

\subsection{Gauged world-sheet action}

In order to follow Buscher's approach to T-duality, we now promote the global symmetries (2.6) to local ones by introducing corresponding gauge fields.

Gauged action I. To gauge the global symmetries, we consider $\epsilon^{\alpha} \equiv \epsilon^{\alpha}\left(\sigma^{a}\right)$ and introduce world-sheet gauge fields $A^{\alpha}$. The resulting gauged action takes the following form

$$
\begin{aligned}
& \widehat{\mathcal{S}}=-\frac{1}{2 \pi \alpha^{\prime}} \int_{\Sigma} {\left[\frac{1}{2} G_{i j}\left(\mathrm{~d} X^{i}+k_{\alpha}^{i} A^{\alpha}\right) \wedge \star\left(\mathrm{d} X^{j}+k_{\beta}^{j} A^{\beta}\right)+\frac{\alpha^{\prime}}{2} \mathrm{R} \phi \star 1\right] } \\
&-\frac{i}{2 \pi \alpha^{\prime}} \int_{\Sigma}\left[\frac{1}{2} B_{i j} \mathrm{~d} X^{i} \wedge \mathrm{d} X^{j}\right.+\left(\tilde{v}_{\alpha}+\mathrm{d} \chi_{\alpha}\right) \wedge A^{\alpha}+\frac{1}{2}\left(\iota_{k_{[\underline{\alpha}}} \tilde{v}_{\beta]}+f_{\left.\left.\alpha \beta^{\gamma} \chi_{\gamma}\right) A^{\alpha} \wedge A^{\beta}\right]}\right. \\
&-\frac{1}{2 \pi \alpha^{\prime}} \int_{\partial \Sigma}\left[2 \pi i \alpha^{\prime} a_{a} \mathrm{~d} X^{a}-i \Omega_{\partial \Sigma}+\alpha^{\prime} k(s) \phi \mathrm{d} s\right],
\end{aligned}
$$

where for later purpose we introduced a set of scalar fields $\chi_{\alpha}$ with $\alpha=1, \ldots, N$ and where we have defined

$$
\tilde{v}_{\alpha}:=v_{\alpha}-\imath_{k_{\alpha}} B
$$

The one-form $\Omega_{\partial \Sigma}$ depends on whether the local symmetries are along Neumann or Dirichlet directions and we specify its precise form below. The local symmetry transformations for 
the gauged action (2.11) are given as follows ${ }^{2}$

$$
\begin{aligned}
& \hat{\delta}_{\epsilon} X^{i}=\epsilon^{\alpha} k_{\alpha}^{i}, \\
& \hat{\delta}_{\epsilon} A^{\alpha}=-\mathrm{d} \epsilon^{\alpha} \quad-f_{\beta \gamma}{ }^{\alpha} \epsilon^{\beta} A^{\gamma}, \\
& \hat{\delta}_{\epsilon} \chi_{\alpha}=-\iota_{k_{(\bar{\alpha}}} v_{\bar{\beta})} \epsilon^{\beta}-f_{\alpha \beta}{ }^{\gamma} \epsilon^{\beta} \chi_{\gamma},
\end{aligned}
$$

however, under (2.13) the gauged action (2.11) is in general not invariant. For invariance of the terms in the bulk $\Sigma$ we have to require that $\mathrm{d} \chi_{\alpha}$ are globally well-defined on $\Sigma$ and have to impose the additional constraints

$$
\mathcal{L}_{k_{[\underline{\alpha}}} \tilde{v}_{\beta]}=f_{\alpha \beta} \tilde{v}_{\gamma}, \quad \quad \iota_{k_{[\underline{\alpha}}} f_{\underline{\beta \gamma}]}^{\delta} \tilde{v}_{\delta}=\frac{1}{3} \iota_{k_{\alpha}} \iota_{k_{\beta}} \iota_{k_{\gamma}} H .
$$

Boundary conditions. Let us now come to the boundary conditions for the gauged action. In particular, the conditions (2.4) for the fields $X^{i}$ are preserved provided that the transformation parameters $\epsilon^{\alpha}$ satisfy on the boundary

$$
\begin{array}{ll}
\text { Dirichlet } & 0=\left.k_{\alpha}^{\hat{i}}\left(\mathrm{~d} \epsilon^{\alpha}\right)_{\tan }\right|_{\partial \Sigma}, \\
\text { Neumann } & 0=G_{a i} k_{\alpha}^{i}\left(\mathrm{~d} \epsilon^{\alpha}\right)_{\text {norm }}+\left.2 \pi \alpha^{\prime} i \mathcal{F}_{a b} k_{\alpha}^{b}\left(\mathrm{~d} \epsilon^{\alpha}\right)_{\tan }\right|_{\partial \Sigma},
\end{array}
$$

where we employed the restrictions (2.10) coming from the global symmetries. However, for Dirichlet boundary conditions we again have a stronger requirement from demanding that $\left.\delta X^{\hat{i}}\right|_{\partial \Sigma}=0$. This implies in particular that $\left.k_{\alpha}^{\hat{i}} \epsilon^{\alpha}\right|_{\partial \Sigma}=0$.

We now turn to the boundary conditions for the gauge fields $A^{\alpha}$ and start with the following two observations:

- For Dirichlet conditions we argued that the corresponding transformation parameters $\epsilon^{\alpha}$ have to vanish on the boundary. Comparing with the transformations (2.13), we can conclude that under local symmetry transformations the gauge fields $A^{\alpha}$ do not change on the boundary. In fact, as we will discuss in section 2.3 , in order to show the equivalence to the ungauged action we demand that $A^{\alpha}$ vanishes on the boundary.

- For Neumann conditions we can determine the equations of motion for $X^{i}$ from the gauged action (2.11). If we require that for this variation boundary terms vanish, we obtain conditions for the $A^{\alpha}$ summarized below.

Motivated by these observations, we then impose the following boundary conditions for the gauge fields $A^{\alpha}[41]$

$$
\begin{array}{ll}
\text { Dirichlet } & 0=\left.k_{\alpha}^{\hat{i}}\left(A^{\alpha}\right)_{\tan }\right|_{\partial \Sigma}, \\
\text { Neumann } & 0=G_{a i} k_{\alpha}^{i}\left(A^{\alpha}\right)_{\text {norm }}+\left.2 \pi \alpha^{\prime} i \mathcal{F}_{a b} k_{\alpha}^{b}\left(A^{\alpha}\right)_{\tan }\right|_{\partial \Sigma} .
\end{array}
$$

Note that these conditions are preserved under the local symmetry transformations (2.13).

\footnotetext{
${ }^{2}$ Our convention for the (anti-)symmetrization of indices contains a factor of $1 / n$ !, and for better distinction we highlight the (anti-)symmetrized indices by under-lining or over-lining them.
} 
Gauged action II. After having discussed the boundary conditions for the gauge fields, we can now specify the one-form $\Omega_{\partial \Sigma}$ in the action (2.11). For simplicity we consider local symmetry transformations either all along Dirichlet directions $X^{\hat{i}}$ or all along Neumann directions $X^{a}$. Mixed cases can also be treated, but the presentation becomes more involved.

- We start with Dirichlet boundary conditions. In this case the infinitesimal variation parameters $\epsilon^{\alpha}$ vanish on the boundary, and hence the boundary terms in (2.11) stay invariant under (2.13). For $\Omega_{\partial \Sigma}$ we then choose

$$
\text { Dirichlet } \quad \Omega_{\partial \Sigma}=0 \text {. }
$$

- For Neumann boundary conditions we introduce a second set of Lagrange multipliers $\phi_{\alpha}$ with $\alpha=1, \ldots, N$, and we specify the one-form $\Omega_{\partial \Sigma}$ as

$$
\text { Neumann } \quad \Omega_{\partial \Sigma}=\left(\chi_{\alpha}+\phi_{\alpha}+\omega_{\alpha}-2 \pi \alpha^{\prime} \iota_{k_{\alpha}} a\right) A^{\alpha} .
$$

The $\phi_{\alpha}$ are required to be constant fields on the boundary $\partial \Sigma$, and the $\chi_{\alpha}$ have to be globally well-defined on the boundary $\partial \Sigma$. The latter requirement implies that $\mathrm{d} \chi_{\alpha}$ is exact on the boundary, and hence it follows from the Hodge decomposition (2.5) that $C c C_{N}$-part of $\mathrm{d} \chi_{\alpha}$ vanishes. We therefore have in summary

$$
\begin{aligned}
& \chi_{\alpha} \ldots \text { globally-defined functions on } \Sigma, \\
& \phi_{\alpha} \ldots \text { constant functions on } \partial \Sigma .
\end{aligned}
$$

Finally, in order for the gauged action (2.11) to be invariant under the symmetry transformations (2.13) in the case of Neumann boundary conditions, in addition to $(2.14)$ we impose

$$
\left.\mathcal{L}_{k_{[\underline{\alpha}}} \omega_{\underline{\beta}]}\right|_{\partial \Sigma}=\left.\frac{1}{2}\left[f_{\alpha \beta} \omega_{\gamma}+\iota_{k_{[\underline{\alpha}}} v_{\underline{\beta}]}\right]\right|_{\partial \Sigma}, \quad 0=\left.f_{\alpha \beta} \phi_{\gamma}\right|_{\partial \Sigma} .
$$

Symmetries of the gauged action. The gauged action (2.11) has been constructed such that it is invariant under the local transformations (2.6). However, by extending the original action by additional fields $v_{\alpha}, \omega_{\alpha}$ and $\phi_{\alpha}$, further symmetries may arise. And indeed, we find the following transformations which leave the action (2.11) invariant [38]:

1. Gauge transformations of the Kalb-Ramond field with a globally well-defined oneform on the world-sheet $\Sigma$ denoted by $\Lambda$ :

$$
\begin{aligned}
& B \rightarrow B+\mathrm{d} \Lambda, \\
& a \rightarrow a-\frac{1}{2 \pi \alpha^{\prime}} \Lambda, \\
& v_{\alpha} \rightarrow v_{\alpha}+\iota_{k_{\alpha}} \mathrm{d} \Lambda, \\
& \omega_{\alpha} \rightarrow \omega_{\alpha}-\iota_{k_{\alpha}} \Lambda .
\end{aligned}
$$


2. Shifts of the one-forms $v_{\alpha}$ by exact forms using functions $\lambda_{\alpha}$ :

$$
\begin{aligned}
v_{\alpha} & \rightarrow v_{\alpha}+\mathrm{d} \lambda_{\alpha}, \\
\chi_{\alpha} & \rightarrow \chi_{\alpha}-\lambda_{\alpha}, \\
\omega_{\alpha} & \rightarrow \omega_{\alpha}+\lambda_{\alpha},
\end{aligned} \quad \mathcal{L}_{k_{[\underline{\alpha}}} \lambda_{\underline{\beta}]}=f_{\alpha \beta} \lambda_{\gamma} .
$$

3. Gauge transformations of the open-string gauge field $a$ with a globally well-defined function $\lambda$ on the boundary $\partial \Sigma$ :

$$
\begin{aligned}
& a \rightarrow a+\mathrm{d} \lambda, \\
& \omega_{\alpha} \rightarrow \omega_{\alpha}+2 \pi \alpha^{\prime} \imath_{k_{\alpha}} \mathrm{d} \lambda .
\end{aligned}
$$

4. Shifts of the functions $\omega_{\alpha}$ by constants $\theta_{\alpha}$ :

$$
\begin{aligned}
& \chi_{\alpha} \rightarrow \chi_{\alpha}+\theta_{\alpha}, \\
& \omega_{\alpha} \rightarrow \omega_{\alpha}-\theta_{\alpha},
\end{aligned} \quad f_{\alpha \beta}^{\gamma} \theta_{\gamma}=0 .
$$

5. Shifts of the functions $\phi_{\alpha}$ by constants $\Theta_{\alpha}$ :

$$
\begin{aligned}
\phi_{\alpha} & \rightarrow \phi_{\alpha}+\Theta_{\alpha}, \\
\omega_{\alpha} & \rightarrow \omega_{\alpha}-\Theta_{\alpha} .
\end{aligned}
$$

Note that for Dirichlet boundary conditions the boundary term $\Omega_{\partial \Sigma}$ vanishes, and therefore the last two symmetries are slightly modified and less restrictive.

\subsection{Recovering the ungauged world-sheet action}

We finally want to show how the ungauged world-sheet theory (2.1) can be recovered from the gauged action (2.11). This will be done using the equations of motion of the Lagrange multipliers $\chi_{\alpha}$ (and $\phi_{\alpha}$ ) [55-57].

Equations of motion for $\chi_{\alpha}$ (and $\phi_{\alpha}$ ). Let us start by determining the equations of motion for the Lagrange multipliers from the gauged action (2.11). We distinguish again between all-Dirichlet or all-Neumann boundary conditions:

- In the case of Dirichlet boundary conditions, we recall from (2.17) that the one-form $\Omega_{\partial \Sigma}$ vanishes. The variation of the action (2.11) with respect to $\chi_{\alpha}$ then leads to

$$
\delta_{\chi} \widehat{\mathcal{S}}=\frac{i}{2 \pi \alpha^{\prime}} \int_{\Sigma} \delta \chi_{\alpha}\left(\mathrm{d} A^{\alpha}-\frac{1}{2} f_{\beta \gamma}{ }^{\alpha} A^{\beta} \wedge A^{\gamma}\right)
$$

where the boundary term vanishes due to the Dirichlet conditions (2.16). Setting to zero the variation (2.26) leads to the equations of motion for $A^{\alpha}$, and together with the boundary condition on $A^{\alpha}$ we have

$$
0=F^{\alpha}=\mathrm{d} A^{\alpha}-\frac{1}{2} f_{\beta \gamma}{ }^{\alpha} A^{\beta} \wedge A^{\gamma}, \quad 0=\left.A^{\alpha}\right|_{\partial \Sigma} .
$$


- Next, we turn to the Neumann boundary conditions. In this case the boundary oneform $\Omega_{\partial \Sigma}$ takes the form given in (2.18), and for the equations of motion for $\chi_{\alpha}$ and $\phi_{\alpha}$ we determine

$$
\begin{aligned}
\delta_{\chi} \widehat{\mathcal{S}} & =\frac{i}{2 \pi \alpha^{\prime}} \int_{\Sigma} \delta \chi_{\alpha}\left(\mathrm{d} A^{\alpha}-\frac{1}{2} f_{\beta \gamma}{ }^{\alpha} A^{\beta} \wedge A^{\gamma}\right), \\
\delta_{\phi} \widehat{\mathcal{S}} & =\frac{i}{2 \pi \alpha^{\prime}} \int_{\partial \Sigma} \delta \phi_{\alpha} A^{\alpha},
\end{aligned}
$$

which gives the equations of motion

$$
0=F^{\alpha}=\mathrm{d} A^{\alpha}-\frac{1}{2} f_{\beta \gamma}{ }^{\alpha} A^{\beta} \wedge A^{\gamma}, \quad 0=\left.A^{\alpha}\right|_{\partial \Sigma} .
$$

Here it also becomes apparent why in the case of Neumann boundary conditions we introduced a second set of Lagrange multipliers $\phi_{\alpha}$. The latter are needed in order to set to zero the gauge field on the boundary [38].

Abelian isometry algebra. Let us now consider abelian isometry algebras for which the structure constants $f_{\alpha \beta} \gamma$ are vanishing. In this case the equation of motion for $\chi_{\alpha}$ imply that $A^{\alpha}$ is closed, and according to (2.5) we can therefore decompose

$$
A^{\alpha}=\mathrm{d} a_{(0)}^{\alpha}+\sum_{\mathrm{m}} a_{(\mathrm{m})}^{\alpha} \varphi^{\mathrm{m}}
$$

where $a_{(0)}^{\alpha}$ are globally defined functions on $\Sigma, a_{(\mathrm{m})}^{\alpha} \in \mathbb{R}$ and $\varphi^{\mathrm{m}} \in C c C_{N}^{1}$ is a basis of closed and co-closed one-forms on $\Sigma$ whose normal part vanishes. However, taking into account also the second condition in (2.27) and (2.29) we see that the tangential part of $A^{\alpha}$ is required to vanish on the boundary. As we discussed before, this implies that the $C c C_{N}^{1}$ part of $A^{\alpha}$ is trivial, i.e. $a_{(\mathrm{m})}^{\alpha}=0$. Therefore $A^{\alpha}$ is exact - and using the gauge symmetry (2.13) we can set $A^{\alpha}$ to zero. We have then recovered the original action (2.1) from the gauged one (2.11).

Non-abelian isometry algebra. In the case of a non-abelian isometry algebra the situation is different. Since due to the first condition in (2.27) and (2.29) the gauge fields $A^{\alpha}$ are not closed, we cannot apply the Hodge decomposition theorem. Heuristically, we can follow a method similar to the one in [58] where a field redefinition from $\mathrm{D} X^{i}=\mathrm{d} X^{i}+k_{\alpha}^{i} A^{\alpha}$ to $\mathrm{d} Y^{i}$ has been discussed. This procedure allows to recover the original action from the gauged action also in the case of non-abelian isometries, however, it does not take into account a non-trivial topology of the world-sheet.

More accurate would be to start from the cohomology of the gauge-covariant derivative and determine a corresponding Hodge decomposition theorem for manifolds with boundary. This is however beyond the scope of this paper.

\section{T-duality}

In this section we discuss collective T-duality transformations for the open string. In section 3.1 we first present results for the closed-string sector of the T-dual theory, whereas in section 3.2 and 3.3 we consider the open-string sector with Neumann and Dirichlet boundary conditions. 


\subsection{Closed-string sector}

We start by determining the metric and Kalb-Ramond $B$-field of the T-dual background. We do so by following Buscher's procedure [34, 59] of gauging target-space isometries as discussed in section 2.2 - and integrating-out the corresponding gauge fields $A^{\alpha}$.

Equations of motion for $\boldsymbol{A}^{\alpha}$. The equations of motion for the gauge fields $A^{\alpha}$ are obtained by varying the gauged action (2.11) with respect to $A^{\alpha}$. Since the latter appear without a derivative, we can solve the equations of motion algebraically. From the part of the action defined on the bulk $\Sigma$ we find using matrix notation [58]

$$
A^{\alpha}=-\left(\left[\mathcal{G}-\mathcal{D} \mathcal{G}^{-1} \mathcal{D}\right]^{-1}\right)^{\alpha \beta}\left(\mathbb{1}+i \star \mathcal{D} \mathcal{G}^{-1}\right)_{\beta}^{\gamma}(\mathrm{k}+i \star \xi)_{\gamma},
$$

where we recall that $\alpha, \beta=1, \ldots, N$. For ease of notation, we have defined the following quantities

$$
\begin{array}{ll}
\mathcal{G}_{\alpha \beta}=k_{\alpha}^{i} G_{i j} k_{\beta}^{j}, & \xi_{\alpha}=\mathrm{d} \chi_{\alpha}+\tilde{v}_{\alpha}, \\
\mathcal{D}_{\alpha \beta}=\iota_{k_{[\underline{\alpha}}} \tilde{v}_{\beta]}+f_{\alpha \beta}{ }^{\gamma} \chi_{\gamma}, & \mathrm{k}_{\alpha}=k_{\alpha}^{i} G_{i j} \mathrm{~d} X^{j} .
\end{array}
$$

Note that although in (3.1) the inverse of the matrix $\mathcal{G}$ appears, in the integrated-out action only the inverse of $(\mathcal{G} \pm \mathcal{D})$ plays a role. We therefore require invertibility only for the latter.

The contribution to the equations of motion for $A^{\alpha}$ from the boundary $\partial \Sigma$ depends on the type of boundary conditions for the gauge fields (2.16). For instance, if we impose Dirichlet conditions the gauge fields are absent on the boundary as shown in (2.17). On the other hand, if $A^{\alpha}$ satisfy Neumann boundary conditions we find a non-trivial condition which has to be imposed as a constraint. In particular, we have

$$
\begin{array}{ll}
\text { Dirichlet } & \varnothing, \\
\text { Neumann } & 0=2 \pi \alpha^{\prime} \iota_{k_{\alpha}} a-\left.\left(\chi_{\alpha}+\phi_{\alpha}+\omega_{\alpha}\right)\right|_{\partial \Sigma} .
\end{array}
$$

Integrated-out action. Using the expressions (3.1) and (3.3), we can now evaluate the action (2.11). We obtain the following general form

$$
\begin{aligned}
\check{\mathcal{S}}= & -\frac{1}{2 \pi \alpha^{\prime}} \int_{\Sigma}\left[\check{G}+i \check{B}+\frac{\alpha^{\prime}}{2} \mathrm{R} \phi \star 1\right] \\
& -\frac{1}{2 \pi \alpha^{\prime}} \int_{\partial \Sigma}\left[2 \pi i \alpha^{\prime} a_{a} \mathrm{~d} X^{a}+\alpha^{\prime} k(s) \phi \mathrm{d} s\right],
\end{aligned}
$$

with the world-sheet quantities $\check{G}$ and $\check{B}$ given by the expressions

$$
\begin{aligned}
& \check{G}=G-\frac{1}{2}(\mathrm{k}+\xi)^{T}(\mathcal{G}+\mathcal{D})^{-1} \wedge \star(\mathrm{k}-\xi), \\
& \check{B}=B-\frac{1}{2}(\mathrm{k}+\xi)^{T}(\mathcal{G}+\mathcal{D})^{-1} \wedge(\mathrm{k}-\xi),
\end{aligned}
$$


in which matrix multiplications for the indices $\alpha, \beta=1, \ldots, N$ is understood. We note that the original metric and $B$-field appearing in (3.5) read $G=\frac{1}{2} G_{i j} \mathrm{~d} X^{i} \wedge \star \mathrm{d} X^{j}$ and $B=\frac{1}{2} B_{i j} \mathrm{~d} X^{i} \wedge \mathrm{d} X^{j}$. The fields in (3.5) can be regarded as a "metric and $B$-field" for an enlarged target space of dimension $D+N$, which is locally parametrized by coordinates $\left\{X^{i}, \chi_{\alpha}\right\}[60]$.

Change of basis. The symmetric matrix $\breve{G}$ defined through (3.5) has $N$ null-eigenvalues. In the basis $\left\{\mathrm{d} X^{i}, \mathrm{~d} \chi_{\alpha}\right\}$ the corresponding $N$ null-eigenvectors are of the form $[58,60]$

$$
\check{n}_{\alpha}=\left(\begin{array}{c}
k_{\alpha}^{i} \\
\mathcal{D}_{\alpha \beta}-\iota_{k_{\alpha}} \tilde{v}_{\beta}
\end{array}\right),
$$

which can be used to perform a change of basis. Since the Killing vectors $k_{\alpha}$ are assumed to be linearly independent, we can always find a coordinate system in which the $N \times N$ matrix $k_{\alpha}^{\beta}$ is invertible and where all other components of $k_{\alpha}^{i}$ vanish. ${ }^{3}$ Let us then define the following basis of one-forms

$$
\begin{aligned}
& e^{\alpha}=\left(k^{-1}\right)_{\beta}^{\alpha} \mathrm{d} X^{\beta}, \\
& e^{m}=\mathrm{d} X^{m}, \\
& e_{\alpha}=\mathrm{d} \chi_{\alpha}+\left[\iota_{k_{(\bar{\alpha}}} v_{\bar{\beta})}+f_{\alpha \beta^{\gamma}} \chi_{\gamma}\right]\left(k^{-1}\right)^{\beta}{ }_{\gamma} \mathrm{d} X^{\gamma},
\end{aligned}
$$

where the indices take values $\alpha, \beta=1, \ldots, N$ and $m, n=N+1, \ldots, D$. We can now express the fields (3.5) in this new basis:

- Since the symmetric two-tensor $\breve{G}$ has $N$ zero-eigenvalues, it can be brought into the form

$$
\check{G}=\frac{1}{2} \check{\mathrm{G}}_{I J} e^{I} \wedge \star e^{J},
$$

where we employed the notation $e^{I}=\left\{e_{\alpha}, e^{m}\right\}$ with $I=1, \ldots, D$. Using the definitions shown in (3.2) and (2.12), the components $\check{G}_{I J}$ take the following form

$$
\begin{aligned}
\check{\mathrm{G}}_{m n}=G_{m n} & -\mathrm{k}_{\alpha m}\left[(\mathcal{G}+\mathcal{D})^{-1} \mathcal{G}(\mathcal{G}-\mathcal{D})^{-1}\right]^{\alpha \beta} \mathrm{k}_{\beta n} \\
& -\mathrm{k}_{\alpha m}\left[(\mathcal{G}+\mathcal{D})^{-1} \mathcal{D}(\mathcal{G}-\mathcal{D})^{-1}\right]^{\alpha \beta} \tilde{v}_{\beta n} \\
& +\tilde{v}_{\alpha m}\left[(\mathcal{G}+\mathcal{D})^{-1} \mathcal{D}(\mathcal{G}-\mathcal{D})^{-1}\right]^{\alpha \beta} \mathrm{k}_{\beta n} \\
& +\tilde{v}_{\alpha m}\left[(\mathcal{G}+\mathcal{D})^{-1} \mathcal{G}(\mathcal{G}-\mathcal{D})^{-1}\right]^{\alpha \beta} \tilde{v}_{\beta n} \\
\check{\mathrm{G}}^{\alpha}{ }_{n}=+\left[(\mathcal{G}+\mathcal{D})^{-1} \mathcal{D}(\mathcal{G}-\mathcal{D})^{-1}\right]^{\alpha \beta} \mathrm{k}_{\beta n} & \\
& +\left[(\mathcal{G}+\mathcal{D})^{-1} \mathcal{G}(\mathcal{G}-\mathcal{D})^{-1}\right]^{\alpha \beta} \tilde{v}_{\beta n} \\
\check{\mathrm{G}}^{\alpha \beta}=+ & \left.+(\mathcal{G}+\mathcal{D})^{-1} \mathcal{G}(\mathcal{G}-\mathcal{D})^{-1}\right]^{\alpha \beta}
\end{aligned}
$$

\footnotetext{
${ }^{3}$ This is true when the isometry group has no fixed points or only isolated fixed points. If however the isotropy of the isometry group is non-trivial, the matrix $k_{\alpha}^{\beta}$ is not invertible [56] and a different basis of one-forms $e^{\alpha}, e^{m}$ and $e_{\alpha}$ in (3.7) has to be chosen.
} 
These expressions are the components of the metric of the dual background after performing a collective T-duality transformation along $N$ directions. In particular, for the case of a T-duality along one direction these formulas reduce to the usual Buscher rules [34].

- For the two-form $\check{B}$ given in (3.5) we find a slightly different structure. In particular, we can write

$$
\check{B}=\frac{1}{2} \check{\mathrm{B}}_{I J} e^{I} \wedge e^{J}+\check{B}^{\text {res. }},
$$

where the anti-symmetric matrix $\check{\mathrm{B}}_{I J}$ takes the form

$$
\begin{aligned}
\check{\mathrm{B}}_{m n}=B_{m n} & +\mathrm{k}_{\alpha m}\left[(\mathcal{G}+\mathcal{D})^{-1} \mathcal{D}(\mathcal{G}-\mathcal{D})^{-1}\right]^{\alpha \beta} \mathrm{k}_{\beta n} \\
& +\mathrm{k}_{\alpha m}\left[(\mathcal{G}+\mathcal{D})^{-1} \mathcal{G}(\mathcal{G}-\mathcal{D})^{-1}\right]^{\alpha \beta} \tilde{v}_{\beta n} \\
& -\tilde{v}_{\alpha m}\left[(\mathcal{G}+\mathcal{D})^{-1} \mathcal{G}(\mathcal{G}-\mathcal{D})^{-1}\right]^{\alpha \beta} \mathrm{k}_{\beta n} \\
& -\tilde{v}_{\alpha m}\left[(\mathcal{G}+\mathcal{D})^{-1} \mathcal{D}(\mathcal{G}-\mathcal{D})^{-1}\right]^{\alpha \beta} \tilde{v}_{\beta n} \\
\check{\mathrm{B}}^{\alpha}{ }_{n}=-\left[(\mathcal{G}+\mathcal{D})^{-1} \mathcal{G}(\mathcal{G}-\mathcal{D})^{-1}\right]^{\alpha \beta} \mathrm{k}_{\beta n} & \\
& -\left[(\mathcal{G}+\mathcal{D})^{-1} \mathcal{D}(\mathcal{G}-\mathcal{D})^{-1}\right]^{\alpha \beta} \tilde{v}_{\beta n} \\
\check{\mathrm{B}}^{\alpha \beta}=- & {\left[(\mathcal{G}+\mathcal{D})^{-1} \mathcal{D}(\mathcal{G}-\mathcal{D})^{-1}\right]^{\alpha \beta} }
\end{aligned}
$$

These expressions give the $B$-field of the T-dual background, which in the case of a single T-duality again match with the Buscher rules.

Let us finally address the residual $B$-field $\check{B}^{\text {res. }}$ mentioned in (3.10). Through the one-forms $e^{\alpha}$ it depends on $\mathrm{d} X^{\alpha}$ of the original background, and it takes the explicit form

$$
\check{B}^{\text {res. }}=e^{\alpha} \wedge\left[\mathrm{d} \chi_{\alpha}+v_{\alpha}+\frac{1}{2}\left(\iota_{k_{\underline{\underline{\alpha}}}} v_{\underline{\beta}]}+f_{\alpha \beta}{ }^{\gamma} \chi_{\gamma}\right) e^{\beta}\right] .
$$

We discuss this expression separately for Neumann and Dirichlet boundary conditions in the following two subsections.

Dilaton. The dual dilaton $\check{\phi}$ has to be determined by a one-loop computation as in [59]. However, here we determine $\check{\phi}$ by demanding that the combination $e^{-2 \phi} \sqrt{\operatorname{det} G}$ is invariant under T-duality transformations. We then find

$$
\check{\phi}=\phi-\frac{1}{4} \frac{\operatorname{det} G}{\operatorname{det} \check{G}} .
$$

Closure of basis. In our above discussion, we have identified $e^{I}=\left\{e_{\alpha}, e^{m}\right\}$ with $I=$ $1, \ldots, D$ as the basis one-forms of the T-dual background. As such, they have to be closed under the exterior derivative $\mathrm{d}$. Let us therefore compute the following expressions

$$
\begin{aligned}
& \mathrm{d} e^{\alpha}=-\frac{1}{2} f_{\beta \gamma}{ }^{\alpha} e^{\beta} \wedge e^{\gamma}-\left(k^{-1}\right)_{\beta}^{\alpha}\left[\partial_{m} k_{\gamma}^{\beta}\right] e^{m} \wedge e^{\gamma}, \\
& \mathrm{d} e^{m}=0, \\
& \mathrm{~d} e_{\alpha}=-f_{\alpha \beta}{ }^{\gamma} e^{\beta} \wedge e_{\gamma}+\left(\partial_{m} \iota_{k_{(\bar{\alpha}}} v_{\bar{\beta})}-\left[\iota_{k_{(\bar{\alpha}}} v_{\bar{\gamma})}+f_{\alpha \gamma}{ }^{\delta} \chi_{\delta}\right]\left(k^{-1}\right)_{\epsilon}^{\gamma}\left[\partial_{m} k_{\beta}^{\epsilon}\right]\right) e^{m} \wedge e^{\beta},
\end{aligned}
$$


from which we see that in general the basis $e^{I}=\left\{e_{\alpha}, e^{m}\right\}$ does not close under the exterior derivative $\mathrm{d}$. This means that the dual background may implicitly depend on the original coordinates, which is a property expected from a non-geometric background. Nevertheless, if we restrict ourselves to either of the following situations

$$
\left\{\begin{array}{l}
0=f_{\alpha \beta}{ }^{\gamma} \\
0=\partial_{m} \iota_{(\bar{\alpha}} v_{\bar{\beta})} \\
0=\partial_{m} k_{\alpha}^{\beta}
\end{array}\right\}, \quad\left\{\begin{array}{l}
0=f_{\alpha \beta} \gamma \\
0=\iota_{k_{(\bar{\alpha}}} v_{\bar{\beta})}
\end{array}\right\},
$$

we see that the basis $e^{I}=\left\{e_{\alpha}, e^{m}\right\}$ is closed under the exterior derivative. Note also that, as mentioned in footnote 3 , in the case of a non-trivial isotropy of the isometry group a different basis of one-forms has to be chosen. In this case the exterior algebra may take a different form.

Remark on non-geometric fluxes. Given the general form of the dual metric and $B$ field after a collective T-duality transformation shown in (3.9) and (3.11), we want to take the opportunity and remark on possible non-geometric fluxes. In particular, given $\check{\mathrm{G}}_{I J}$ and $\check{\mathrm{B}}_{I J}$ we can define a new metric $\mathrm{g}^{I J}$ and bi-vector field $\beta^{I J}$ via

$$
(\check{\mathrm{G}} \pm \check{\mathrm{B}})^{-1}=\mathrm{g} \pm \beta
$$

where $\mathrm{g}^{I J}$ corresponds to the symmetric part and $\beta^{I J}$ to the anti-symmetric part. The non-geometric $Q$ - and $R$-fluxes are then expressed in terms of $\beta$ as follows

$$
Q_{I}^{J K}=\partial_{I} \beta^{J K}, \quad R^{I J K}=3 \beta^{[\underline{I M}} \partial_{M} \beta^{J K]} .
$$

Let us now consider a $D$-dimensional background and perform a collective T-duality transformation along all $D$ directions. Ignoring for a moment that the basis of dual oneforms does not close among itself under d, from (3.9) and (3.11) we can determine

$$
\mathrm{g}_{\alpha \beta}=\mathcal{G}_{\alpha \beta}, \quad \beta_{\alpha \beta}=\mathcal{D}_{\alpha \beta},
$$

where we note that for the dual coordinates $\chi_{\alpha}$ the position of the index is reversed as compared to the original coordinates $X^{\alpha}$. For the non-geometric fluxes we compute using (2.14) and the Jacobi identity of $f_{\alpha \beta}{ }^{\gamma}$

$$
Q_{\beta \gamma}^{\alpha}=f_{\beta \gamma}^{\alpha}, \quad R_{\alpha \beta \gamma}=\iota_{k_{\alpha}} \iota_{k_{\beta}} \iota_{k_{\gamma}} H .
$$

The general form of these expressions is as expected: for a background with a non-abelian isometry group the metric is usually non-trivial and one expects a corresponding nongeometric flux related to $f_{\alpha \beta}{ }^{\gamma}$. Under a collective T-duality transformation along all directions this geometric flux should be mapped into the non-geometric $Q$-flux, as in (3.19). Furthermore, under a collective T-duality transformation along all directions the $H$-flux is expected to be mapped into the non-geometric $R$-flux, as can be seen in (3.19). However, 
as mentioned above, the dual-basis one-forms $e_{\alpha}$ defined in (3.7) do not closed under the exterior derivative

$$
\mathrm{d} e_{\alpha}=-f_{\alpha \beta} e^{\beta} \wedge e_{\gamma} .
$$

In this way the dual geometry implicitly depends on the original coordinates, which on general grounds is expected from a non-geometric background.

\subsection{Open-string sector - Neumann directions}

Let us now consider the open-string sector and specialize to T-duality transformations along multiple Neumann directions $X^{a}$. T-duality along Dirichlet directions will be discussed in section 3.3, but the mixed case of collective T-duality along Neumann and Dirichlet directions at the same time will not be studied separately.

Integrating-out I - gauge fields $\boldsymbol{A}^{\alpha}$. We start by integrating-out the gauge fields $A^{\alpha}$ from the gauged action (2.11), taking into account the Neumann boundary conditions shown in (2.16). The solution to the equations of motion for $A^{\alpha}$ in $\Sigma$ has been given in equation (3.1), which leads to the dual metric and $B$-field shown in (3.9) and (3.11), including the residual $B$-field (3.12). The contribution to the equation of motion for $A^{\alpha}$ coming from the boundary leads to the constraint (3.3), which we implement as a $\delta$-function into the path integral

$$
\delta\left(\phi_{\alpha}-\tilde{\chi}_{\alpha}\right)_{\partial \Sigma}, \quad \tilde{\chi}_{\alpha}=\chi_{\alpha}+\omega_{\alpha}-2 \pi \alpha^{\prime} \iota_{k_{\alpha}} a .
$$

Furthermore, the gauge fields $A^{\alpha}$ are subject to the Neumann boundary conditions. Evaluating these for the solution (3.1) gives the following general condition on the boundary

$$
0=\left[\left.G_{a i} k_{\alpha}^{i} \star A^{\alpha}\right|_{(3.1)}+\left.2 \pi \alpha^{\prime} i \mathcal{F}_{a b} k_{\alpha}^{b} A^{\alpha}\right|_{(3.1)}\right]_{\partial \Sigma},
$$

where $\left.A^{\beta}\right|_{(3.1)}$ denotes the solution (3.1). In general (3.22) will take a complicated form and has to be computed in a case-by-case analysis. However, if we restrict ourselves for a moment to the abelian situation with $f_{\alpha \beta}{ }^{\gamma}=0$ and contract (3.22) with $k_{\beta}$, we find that

$$
0=\left.\mathrm{d} \tilde{\chi}_{\alpha}\right|_{\partial \Sigma}
$$

These relations describe Dirichlet boundary conditions for the dual coordinates $\chi_{\alpha}$, which we expect on general grounds.

Integrating-out II - Lagrange multipliers $\phi_{\alpha}$. Next, we consider the Lagrange multipliers $\phi_{\alpha}$. After integrating-out the gauge fields $A^{\alpha}$ and implementing the constraint (3.21), the path integral takes the following schematic form

$$
\mathcal{Z}=\int \frac{\left[\mathcal{D} X^{i}\right]\left[\mathcal{D} \chi_{\alpha}\right]}{\mathcal{V}_{\text {gauge }}} \int\left[\mathcal{D} \phi_{\alpha}\right] \delta\left(\phi_{\alpha}-\tilde{\chi}_{\alpha}\right)_{\partial \Sigma} \exp \left(\check{\mathcal{S}}\left[X^{i}, \chi_{\alpha}\right]\right)
$$

where $\mathcal{V}_{\text {gauge }}$ denotes the volume of the local gauge symmetry $(2.13), \tilde{\chi}_{\alpha}$ have been defined in (3.21) and $\check{\mathcal{S}}$ denotes the action (3.4). Since the latter does not depend on $\phi_{\alpha}$, the integral over $\phi_{\alpha}$ can performed trivially and the $\delta$-function (3.21) gives one. 
Integrating-out III - coordinates $\boldsymbol{X}^{\boldsymbol{\alpha}}$. The action $\check{\mathcal{S}}$ still depends on the original coordinates $X^{\alpha}$ which satisfy Neumann boundary conditions. This means in particular that $\mathrm{d} X^{\alpha}$ can have a non-vanishing $C c C_{N}^{1}$-part, so the local symmetry (2.13) cannot be used to set $X^{\alpha}$ to zero. However, the residual $B$-field (3.12) provides the required terms. In the following we restrict ourselves again to the abelian situation and make the technical assumption that $k_{\alpha}^{\beta}$ are constant, but more general cases can be treated in a similar fashion.

To start, let us note that in (2.8) we have shown conditions which relate the openstring gauge field $a$, the one-forms $v_{\alpha}$ and the functions $\omega_{\alpha}$ on the boundary $\partial \Sigma$ to each other. All these quantities depend only on $X^{i}$, which have a unique continuation from the boundary $\partial \Sigma$ to the bulk $\Sigma$. We can therefore assume that the relations (2.8) and (2.14) are valid also on $\Sigma$. This allows us to rewrite $\check{B}^{\text {res. }}$ in the following way

$$
\check{B}^{\text {res. }}=\mathrm{d}\left[-\tilde{\chi}_{\alpha} e^{\alpha}-2 \pi \alpha^{\prime} a\right]+2 \pi \alpha^{\prime}\left(\frac{1}{2} F_{m n} e^{m} \wedge e^{n}\right),
$$

with $F=\mathrm{d} a$ the open-string field strength and $\tilde{\chi}_{\alpha}$ were defined in (3.21). The dual action (3.4) contains $\check{B}^{\text {res. }}$ together with the open-string gauge field on the boundary. For those we compute

$$
\begin{aligned}
& -\frac{i}{2 \pi \alpha^{\prime}} \int_{\Sigma} \check{B}^{\mathrm{res} .}-\frac{i}{2 \pi \alpha^{\prime}} \int_{\partial \Sigma} 2 \pi \alpha^{\prime} a \\
= & +\frac{i}{2 \pi \alpha^{\prime}} \int_{\partial \Sigma} \tilde{\chi}_{\alpha} e^{\alpha}-\frac{i}{2 \pi \alpha^{\prime}} \int_{\Sigma} 2 \pi \alpha^{\prime}\left(\frac{1}{2} F_{m n} e^{m} \wedge e^{n}\right) .
\end{aligned}
$$

The second term in (3.26) denotes the open-string field strength along the directions which are not dualized and combines with $B_{m n}$ in (3.11) into the gauge-invariant open-string field strength. Turning to the first term in (3.26), since the $\mathrm{d} X^{\alpha}$ with Neumann boundary conditions appearing in $e^{\alpha}$ are closed, we can expand them into an exact and a $C c C_{N}^{1}$-part similarly as in equation $(2.30)$

$$
\mathrm{d} X^{\alpha}=\mathrm{d} X_{(0)}^{\alpha}+\sum_{\mathrm{m}} X_{(\mathrm{m})}^{\alpha} \varphi^{\mathrm{m}}
$$

where $X_{(0)}^{\alpha}$ are globally-defined functions on $\Sigma, X_{(\mathrm{m})}^{\alpha} \in \mathbb{R}$ are constants and $\varphi^{\mathrm{m}} \in C c C_{N}^{1}$ is a basis of closed and co-closed one-forms with vanishing normal part. A corresponding basis of the first homology on $\partial \Sigma$ will be denoted by $\gamma_{m}$ and can be normalized as $\int_{\gamma_{m}} \varphi^{n}=\delta_{m}^{n}$. Now, the exact part $X_{(0)}^{\alpha}$ appearing in (3.27) can be set to zero using the symmetry (2.13), while for the $C c C_{N}^{1}$-part we distinguish the following two situations:

- Let us first assume that we can define winding/momentum numbers $n_{(\mathrm{m})}$ for the $X^{\alpha}$ as follows

$$
\oint_{\gamma_{\mathrm{m}}} \mathrm{d} X^{\alpha}=X_{(\mathrm{m})}^{\alpha}=2 \pi n_{(\mathrm{m})}^{\alpha}, \quad \quad n_{(\mathrm{m})}^{\alpha} \in \mathbb{Z}
$$

which determine the $X_{(\mathrm{m})}^{\alpha}$ appearing in (3.27). For a compactification of $X^{\alpha}$ on a circle or a flat torus without $H$-flux these momentum/winding sectors always exist, but on more general backgrounds these may be either absent or not be quantized. 
Coming now back to the first expression in (3.26), we see that the path integral (3.24) (after integrating over $\phi_{\alpha}$ ) contains the following terms

$$
\begin{aligned}
\mathcal{Z} & \supset \int \frac{\left[\mathcal{D} X^{\alpha}\right]}{\mathcal{V}_{\text {gauge }}} \exp \left[\frac{i}{2 \pi \alpha^{\prime}} \int_{\partial \Sigma} \tilde{\chi}_{\alpha} e^{\alpha}\right] \\
& \supset \int \frac{\left[\mathcal{D} X_{(0)}^{\alpha}\right]}{\mathcal{V}_{\text {gauge }}} \sum_{n_{(\mathrm{m})}^{\alpha} \in \mathbb{Z}} \exp \left[\frac{i}{2 \pi \alpha^{\prime}} \int_{\partial \Sigma} \tilde{\chi}_{\beta}\left(k^{-1}\right)_{\alpha}^{\beta} d X^{\alpha}\right] \\
& \supset \sum_{n_{(\mathrm{m})}^{\alpha} \in \mathbb{Z}} \exp \left[\frac{i}{\alpha^{\prime}} \tilde{\chi}_{\beta}\left(k^{-1}\right)_{\alpha}^{\beta} n_{(\mathrm{m})}^{\alpha}\right]_{\partial \Sigma} \\
& \supset \sum_{m_{\alpha(\mathrm{m})} \in \mathbb{Z}} \delta\left[\frac{1}{2 \pi \alpha^{\prime}} \tilde{\chi}_{\beta}\left(k^{-1}\right)_{\alpha}^{\beta}-m_{\alpha(\mathrm{m})}\right]_{\partial \Sigma},
\end{aligned}
$$

where from the second to the third line we set to zero the exact part using the local symmetries, and from the third to the fourth line we employed the definition of the periodic Kronecker $\delta$-symbol [55]. We therefore see that coordinates $\tilde{\chi}_{\alpha}$ on the boundary $\partial \Sigma$ are quantized as

$$
\left.\frac{1}{\alpha^{\prime}} \tilde{\chi}_{\beta}\left(k^{-1}\right)_{\alpha}^{\beta}\right|_{\partial \Sigma} \in 2 \pi \mathbb{Z}
$$

- The second possibility is that $X_{(\mathrm{m})}^{\alpha}$ appearing in (3.27) are real numbers determined via the equations of motion (2.2), which are in particular not quantized. In this case the sum in (3.29) is replaced by integrals over $X_{(\mathrm{m})}^{\alpha}$, leading to Dirac $\delta$-functions which set

$$
\left.\tilde{\chi}_{\alpha}\right|_{\partial \Sigma}=0
$$

Let us also note that $\tilde{\chi}_{\alpha}$ defined in (3.21) contain a contribution from the open-string gauge field $a$. This means that a non-vanishing $\iota_{k_{\alpha}} a$ leads to a shift of the dual coordinates $\chi_{\alpha}$, which is again expected on general grounds.

Summary. Let us summarize the main steps to obtain the dual open-string sector in the case of Neumann boundary conditions. We illustrated this procedure with an abelian isometry algebra with constant Killing vectors, but more general configurations (subject to the questions discussed above) follow a similar pattern:

1. First, we integrate-out the gauge fields $A^{\alpha}$ from the gauged action (2.11). This gives the dual metric and $B$-field in the bulk and imposes the constraint (3.21) on the boundary. The boundary conditions for $A^{\alpha}$ shown in (3.22) imply that the dual coordinates satisfy Dirichlet boundary conditions.

2. Next, we integrate over the Lagrange multipliers $\phi_{\alpha}$ in the path integral. Due to the $\delta$-function (3.21) this integral gives one. 
3. Finally, we perform the path integral over the original coordinates $X^{\alpha}$. The exact part of $\mathrm{d} X^{\alpha}$ can be gauged to zero using the local symmetry (2.13), while the cohomologically non-trivial part of $\mathrm{d} X^{\alpha}$ appears in the residual $B$-field (3.12). The latter either gives rise to a periodic Kronecker $\delta$-symbol in the path integral leading to quantization conditions for the dual coordinates on the boundary, or gives a Dirac $\delta$-function which imposes Dirichlet conditions for the dual coordinates.

We want to point-out that these results are in agreement with the well-known CFT analysis of T-duality for the open string: T-duality along a Neumann direction results in a dual Dirichlet direction, and a non-trivial Wilson line leads to a shift of the dual coordinates on the boundary.

\subsection{Open-string sector - Dirichlet directions}

We now turn to collective T-duality transformations along directions with Dirichlet boundary conditions. Due to the absence of the Lagrange multipliers $\phi_{\alpha}$, the procedure differs slightly from the Neumann case.

Integrating-out $\mathbf{I}$ - gauge fields $\boldsymbol{A}^{\alpha}$. We start again by integrating-out the gauge fields $A^{\alpha}$ from the gauged action (2.11). The equations of motion for $A^{\alpha}$ in the bulk $\Sigma$ lead to the solution (3.1), which in turn gives the dual metric and $B$-field shown in (3.9) and (3.11). The residual $B$-field can be found in (3.12) and - as already summarized in (3.3) - there are no additional conditions arising from the variation of the action with respect to $A^{\alpha}$ on the boundary.

The boundary conditions for $A^{\alpha}$ shown in (2.16) require the gauge fields to vanish on the boundary. For the solution (3.1) this implies in particular that

$$
0=\left[\left.A^{\alpha}\right|_{(3.1)}\right]_{\partial \Sigma}
$$

which using (3.1) and the basis $e^{I}=\left\{e_{\alpha}, e^{m}\right\}$ given in (3.7) can be expressed as

$$
0=\check{\mathrm{G}}_{I}^{\alpha}\left(e^{I}\right)_{\mathrm{norm}}+i \check{\mathrm{B}}^{\alpha}{ }_{I}\left(e^{I}\right)_{\tan } .
$$

By comparing (3.33) with (2.4) we conclude that these relations describe Neumann boundary conditions for the dual coordinates, which is again expected on general grounds.

Integrating-out II - coordinates $\boldsymbol{X}^{\boldsymbol{\alpha}}$. Next, we turn to the original coordinates $X^{\alpha}$ which appear in the action via the residual $B$-field. In the following we assume for simplicity that the Killing vectors are constant and that $v_{\alpha}=0$, but for more general configurations can be treated in a similar way.

Since the original coordinates $X^{\alpha}$ satisfy Dirichlet boundary conditions, the one-forms $\mathrm{d} X^{\alpha}$ are exact on $\Sigma$. This allows us to rewrite the residual $B$-field (3.12) in the following way

$$
\check{B}^{\text {res. }}=e^{\alpha} \wedge \mathrm{d} \chi_{\alpha}=\mathrm{d}\left[X^{\alpha}\left(k^{-1}\right)_{\alpha}^{\beta} e_{\beta}\right]
$$


which for the action implies

$$
-\frac{i}{2 \pi \alpha^{\prime}} \int_{\Sigma} \check{B}^{\text {res. }}=-\frac{i}{2 \pi \alpha^{\prime}} \int_{\partial \Sigma} 2 \pi \alpha^{\prime}\left[\frac{X^{\alpha}\left(k^{-1}\right)_{\alpha}^{\beta}}{2 \pi \alpha^{\prime}} e_{\beta}\right] .
$$

We therefore see that the position of the D-brane in the original theory $\left.X^{\alpha}\right|_{\partial \Sigma}$ determines a constant gauge field for the T-dual theory

$$
\check{\mathrm{a}}^{\alpha}=\left.\frac{1}{2 \pi \alpha^{\prime}}\left(k^{-1}\right)_{\beta}^{\alpha} X^{\beta}\right|_{\partial \Sigma} .
$$

Using the local gauge symmetry (2.13) we can then fix $X^{\alpha}$ in the bulk $\Sigma$ to a convenient value, and trivially perform the corresponding integration in the path integral. In this way we have then obtained the T-dual theory.

Summary. Let us briefly summarize the main steps for obtaining the dual background for a collective T-duality transformation along Dirichlet directions.

1. We first integrate-out the gauge fields $A^{\alpha}$ form the gauged action (2.11) and obtain the dual metric and $B$-field. The boundary conditions (3.32) for the gauge fields then lead to Neumann boundary conditions for the dual coordinates.

2. In contrast to the case of T-duality transformations along Neumann directions, in the present situation there are no Lagrange multipliers $\phi_{\alpha}$ present.

3. In the case of an abelian isometry algebra and vanishing one-forms $v_{\alpha}$, we can rewrite the residual $B$-field (3.12). The latter then leads to a Wilson line along the dual directions determined by the position of the original D-brane. Since the original one-forms $\mathrm{d} X^{\alpha}$ are exact, we can use the local gauge symmetry to fix them to a convenient value in the bulk $\Sigma$ (subject to the boundary conditions on $\partial \Sigma$ ).

We want to point-out again that these results agree with the results expected from a CFT analysis on a background with constant metric and $B$-field. In particular, a T-duality along a Dirichlet direction leads to a Neumann boundary condition, and the position of the original D-brane corresponds to a constant gauge field in the dual theory.

\section{Examples - three-torus with $H$-flux}

In this section we want to illustrate the formalism introduced above with the example of the three-torus with $H$-flux. We discuss a number of different settings and show explicitly that the results expected from toroidal compactifications with constant $B$-field are obtained also for non-trivial $B$-field.

Setup. As a starting point we consider the background of a flat three-torus with $H$-flux and different types of D-branes. We denote coordinates on the three-torus $\mathbb{T}^{3}$ by $X^{i}$ with 
$i=1,2,3$, and impose the identifications $X^{i} \sim X^{i}+2 \pi$. The corresponding basis for the co-tangent space is given by one-forms $\mathrm{d} X^{i}$, and the metric and $B$-field read

$$
G_{i j}=\left(\begin{array}{ccc}
R_{1}^{2} & 0 & 0 \\
0 & R_{2}^{2} & 0 \\
0 & 0 & R_{3}^{2}
\end{array}\right), \quad B=\frac{\alpha^{\prime}}{2 \pi} h X^{3} \mathrm{~d} X^{1} \wedge \mathrm{d} X^{2}, \quad \phi=\phi_{0},
$$

where $h \in \mathbb{Z}$ due to the flux-quantization condition. The radii $R_{i}$ have the dimension of the string-length $\ell_{\mathrm{s}}$, whereas the coordinates $X^{i}$ are dimensionless. The dilaton $\phi$ is taken to be constant, and the Killing vectors we are interested-in (in a basis dual to $\mathrm{d} X^{i}$ ) are given by

$$
k_{1}=\left(\begin{array}{l}
1 \\
0 \\
0
\end{array}\right), \quad k_{2}=\left(\begin{array}{l}
0 \\
1 \\
0
\end{array}\right), \quad k_{3}=\left(\begin{array}{l}
0 \\
0 \\
1
\end{array}\right)
$$

which satisfy an abelian isometry algebra. Our conventions for the open-string sector is that a $\mathrm{D} p$-brane has Neumann boundary conditions along the time direction and along $p$ spatial directions in $\mathbb{T}^{3}$, while the remaining directions are of Dirichlet type. Finally, since in our convention the coordinates $X^{i}$ are dimensionless it turns out to be convenient to use also dimensionless dual coordinates

\subsection{One T-duality}

$$
\check{\chi}_{\alpha}=\frac{1}{\alpha^{\prime}} \chi_{\alpha}
$$

We start with discussing one T-duality transformation for the above background. For convenience we always take the direction $X^{1}$, but the formalism introduced in section 3 gives similar results for the other directions. In particular, we can equally perform a Tduality transformation along the direction $X^{3}$.

D1-brane along $\boldsymbol{X}^{\mathbf{1}}$. Let us place a D1-brane along the direction $X^{1}$ and consider a constant open-string gauge field $a$. The corresponding field strength $F=\mathrm{d} a$ vanishes, and the boundary conditions (2.4) take the form

$$
0=\left(\mathrm{d} X^{1}\right)_{\text {norm }}, \quad 0=\left(\mathrm{d} X^{2}\right)_{\tan }, \quad 0=\left(\mathrm{d} X^{3}\right)_{\tan } .
$$

The constraints (2.8) and (2.14) for a T-duality along the $X^{1}$-direction are solved for instance by

$$
\begin{aligned}
a & =a_{1} \mathrm{~d} X^{1}, \\
v_{1} & =0, \\
\omega_{1} & =0,
\end{aligned} \quad a_{1}=\text { const. },
$$

and the dual metric and $B$-field can be determined from the general expressions (3.9) and (3.11) (together with (4.3)) as

$$
\check{\mathrm{G}}_{I J}=\left(\begin{array}{ccc}
\frac{\alpha^{\prime 2}}{R_{1}^{2}} & -\frac{\alpha^{\prime 2}}{R_{1}^{2}} \frac{h}{2 \pi} X^{3} & 0 \\
-\frac{\alpha^{\prime 2}}{R_{1}^{2}} \frac{h}{2 \pi} X^{3} & R_{2}^{2}+\frac{\alpha^{\prime 2}}{R_{1}^{2}}\left[\frac{h}{2 \pi} X^{3}\right]^{2} & 0 \\
0 & 0 & R_{3}^{2}
\end{array}\right), \quad \check{\mathrm{B}}_{I J}=0 .
$$


This background is known as a twisted three-torus [3, 4], and the dual basis can be read-off from (3.7) as $\left\{\mathrm{d} \check{\chi}_{1}, \mathrm{~d} X^{2}, \mathrm{~d} X^{3}\right\}$. The boundary condition for $\mathrm{d} \check{\chi}_{1}$ is determined via (3.22), and together with the remaining directions we have

$$
0=\left(\mathrm{d} \check{\chi}_{1}\right)_{\tan }, \quad 0=\left(\mathrm{d} X^{2}\right)_{\tan }, \quad 0=\left(\mathrm{d} X^{3}\right)_{\tan } .
$$

The dual background therefore contains a D0-brane. The residual $B$-field (3.12) can be determined as $\check{B}^{\text {res. }}=\mathrm{d} X^{1} \wedge \mathrm{d} \chi_{1}$, and by performing the path integral over $X^{1}$ gives the condition

$$
\left[\check{\chi}_{1}-2 \pi a_{1}\right]_{\partial \Sigma} \in 2 \pi \mathbb{Z}
$$

However, since we do not know how to quantize the theory in the presence of a non-trivial $H$-flux we have no information about the momentum/winding numbers of the original coordinate $X^{1}$. Strictly speaking we should therefore set the right-hand side of (4.8) to zero following (3.31). In summary, we see that the dual background is a twisted torus with a D0-brane, whose position is specified by the Wilson line $a_{1}$.

D2-brane along $\boldsymbol{X}^{\mathbf{1}}-\boldsymbol{X}^{\mathbf{2}}$. As a second example, we consider a D2-brane along the directions $X^{1}$ and $X^{2}$ with a non-trivial open-string field strength $F_{12}=f=$ const. The boundary conditions (2.4) then take the form

$$
\begin{aligned}
& 0=R_{1}^{2}\left(\mathrm{~d} X^{1}\right)_{\text {norm }}+2 \pi \alpha^{\prime} i\left(f+\frac{h}{4 \pi^{2}} X^{3}\right)\left(\mathrm{d} X^{2}\right)_{\tan }, \\
& 0=R_{2}^{2}\left(\mathrm{~d} X^{2}\right)_{\text {norm }}-2 \pi \alpha^{\prime} i\left(f+\frac{h}{4 \pi^{2}} X^{3}\right)\left(\mathrm{d} X^{1}\right)_{\tan }, \\
& 0=\left(\mathrm{d} X^{3}\right)_{\tan },
\end{aligned}
$$

and for a T-duality along the direction $X^{1}$ the constraints (2.8) and (2.14) are solved by

$$
\begin{aligned}
& a=a_{1} \mathrm{~d} X^{1}+a_{2} \mathrm{~d} X^{2}+f X^{1} \mathrm{~d} X^{2}, \\
& v_{1}=-2 \pi \alpha^{\prime} f \mathrm{~d} X^{2}, \\
& \omega_{1}=0
\end{aligned} \quad a_{1}, a_{2}, f=\text { const. }
$$

The T-dual metric and $B$-field are again determined from the general expressions (3.9) and (3.11) with (4.3), from which we find

$$
\begin{aligned}
& \check{\mathrm{G}}_{I J}=\left(\begin{array}{ccc}
\frac{\alpha^{\prime 2}}{R_{1}^{2}} & -\frac{\alpha^{\prime 2}}{R_{1}^{2}}\left[2 \pi f+\frac{h}{2 \pi} X^{3}\right] & 0 \\
-\frac{\alpha^{\prime 2}}{R_{1}^{2}}\left[2 \pi f+\frac{h}{2 \pi} X^{3}\right] & R_{2}^{2}+\frac{\alpha^{\prime 2}}{R_{1}^{2}}\left[2 \pi f+\frac{h}{2 \pi} X^{3}\right]^{2} & 0 \\
0 & 0 & R_{3}^{2}
\end{array}\right), \\
& \check{\mathrm{B}}_{I J}=0,
\end{aligned}
$$

which again describes a twisted three-torus. Note however that here the gauge-invariant open-string field strength $2 \pi \alpha^{\prime} \mathcal{F}_{12}=2 \pi \alpha^{\prime} f+\frac{\alpha^{\prime}}{2 \pi} h X^{3}$ appears. The dual basis is determined 
via (3.7) and reads as before $\left\{\mathrm{d} \check{\chi}_{1}, \mathrm{~d} X^{2}, \mathrm{~d} X^{3}\right\}$, and from (3.22) we find the boundary conditions

$$
\begin{aligned}
& 0=\left(\mathrm{d} \check{\chi}_{1}\right)_{\tan }, \\
& 0=\check{\mathrm{G}}_{2}{ }^{1}\left(\mathrm{~d} \check{\chi}_{1}\right)_{\text {norm }}+\check{\mathrm{G}}_{22}\left(\mathrm{~d} X^{2}\right)_{\text {norm }}, \\
& 0=\left(\mathrm{d} X^{3}\right)_{\tan } .
\end{aligned}
$$

These describe Dirichlet conditions for $\check{\chi}_{1}$ and $X^{3}$ and a Neumann boundary condition for $X^{2}$, and hence the dual backgrounds contains a D1-brane along the $X^{2}$-direction. The residual $B$-field (3.12) is determined as $\check{B}^{\text {res. }}=\mathrm{d} X^{1} \wedge\left(\mathrm{d} \chi_{1}-2 \pi \alpha^{\prime} f \mathrm{~d} X^{2}\right)$, which via (3.26) cancels the open-string gauge field $f X^{1} \mathrm{~d} X^{2} \subset a$ on the boundary. Performing then the path integral over $X^{1}$ gives

$$
\left[\check{\chi}_{1}-2 \pi a_{1}\right]_{\partial \Sigma}=0
$$

following the same reasoning leading to (3.31). The Wilson line $a_{2} \mathrm{~d} X^{2} \subset a$ is untouched, so the dual open-string gauge field reads

$$
\check{\mathrm{a}}=a_{2} \mathrm{~d} X^{2} .
$$

In summary, we find that the T-dual background is a twisted torus with a D1-brane with constant Wilson line along the $X^{2}$-direction. We also note that when turning-off the $H$-flux and setting $h=0$, the metric (4.11) becomes constant. The boundary conditions (4.12) then describe a D1-brane at an angle in the $X^{1}-X^{2}$ torus, which reproduces the well-known CFT result.

D3-brane along $\boldsymbol{X}^{\mathbf{1}}-\boldsymbol{X}^{\mathbf{2}}-\boldsymbol{X}^{\mathbf{3}}$. Let us also briefly discuss a D3-brane along all directions of the three-torus. Such a configuration does not satisfy the Freed-Witten anomaly cancellation condition [61], which says that $H$ pulled-back to the D-brane has to vanish in cohomology. Nevertheless, we perform a T-duality transformation along the $X^{1}$-direction in order to gain insight on the dual background.

For simplicity, we consider a setting similar to the above-discussed D2-brane along $X^{1}-X^{2}$ but replace the Dirichlet boundary conditions along $X^{3}$ by Neumann boundary conditions and set $a=0$. The dual background is then given by

$$
\check{\mathrm{G}}_{I J}=\left(\begin{array}{ccc}
\frac{\alpha^{\prime 2}}{R_{1}^{2}} & -\frac{\alpha^{\prime 2}}{R_{1}^{2}} \frac{h}{2 \pi} X^{3} & 0 \\
-\frac{\alpha^{\prime 2}}{R_{1}^{2}} \frac{h}{2 \pi} X^{3} & R_{2}^{2}+\frac{\alpha^{\prime 2}}{R_{1}^{2}}\left[\frac{h}{2 \pi} X^{3}\right]^{2} & 0 \\
0 & 0 & R_{3}^{2}
\end{array}\right), \quad \check{\mathrm{B}}_{I J}=0
$$

with boundary conditions

$$
\begin{aligned}
& 0=\left(\mathrm{d} \check{\chi}_{1}\right)_{\tan }, \\
& 0=\check{\mathrm{G}}_{2}{ }^{1}\left(\mathrm{~d} \check{\chi}_{1}\right)_{\text {norm }}+\check{\mathrm{G}}_{22}\left(\mathrm{~d} X^{2}\right)_{\text {norm }}, \\
& 0=\left(\mathrm{d} X^{3}\right)_{\text {norm }} .
\end{aligned}
$$


These relations describe a D2-brane with Dirichlet boundary conditions along $\chi_{1}$ and Neumann conditions along $X^{2}$ and $X^{3}$. Since the original configuration is inconsistent, this T-dual configuration has to be inconsistent as well. We come back to this point in section 5.1 .

D0-brane. We also want to illustrate T-duality transformations along Dirichlet directions. To do so, we first consider a D0-brane which is point-like on the three torus. The boundary conditions therefore read

$$
0=\left(\mathrm{d} X^{1}\right)_{\tan }, \quad 0=\left(\mathrm{d} X^{2}\right)_{\tan }, \quad 0=\left(\mathrm{d} X^{3}\right)_{\tan },
$$

and the constraints (2.8) are solved by

$$
a=0, \quad v_{1}=0, \quad \omega_{1}=0 .
$$

The dual background is then given by (3.9) and (3.11) as

$$
\check{\mathrm{G}}_{I J}=\left(\begin{array}{ccc}
\frac{\alpha^{\prime 2}}{R_{1}^{2}} & -\frac{\alpha^{\prime 2}}{R_{1}^{2}} \frac{h}{2 \pi} X^{3} & 0 \\
-\frac{\alpha^{\prime 2}}{R_{1}^{2}} \frac{h}{2 \pi} X^{3} & R_{2}^{2}+\frac{\alpha^{\prime 2}}{R_{1}^{2}}\left[\frac{h}{2 \pi} X^{3}\right]^{2} & 0 \\
0 & 0 & R_{3}^{2}
\end{array}\right), \quad \check{\mathrm{B}}_{I J}=0
$$

which describes again a twisted three-torus. The dual basis is determined via (3.7) and reads $\left\{\mathrm{d} \check{\chi}_{1}, \mathrm{~d} X^{2}, \mathrm{~d} X^{3}\right\}$, which satisfy the boundary conditions

$$
\begin{aligned}
& 0=\check{\mathrm{G}}^{11}\left(\mathrm{~d} \check{\chi}_{1}\right)_{\text {norm }}+\check{\mathrm{G}}_{2}{ }_{2}\left(\mathrm{~d} X^{2}\right)_{\text {norm }}, \\
& 0=\left(\mathrm{d} X^{2}\right)_{\tan }, \\
& 0=\left(\mathrm{d} X^{3}\right)_{\tan } .
\end{aligned}
$$

These expressions describe a Neumann condition for the direction $\check{\chi}_{1}$ and Dirichlet boundary conditions for $X^{2}$ and $X^{3}$, and hence the dual background contains a D1-brane. The residual $B$-field (3.12) takes the form $\check{B}^{\text {res. }}=\mathrm{d} X^{1} \wedge \mathrm{d} \chi_{1}$, and since $X^{1}$ satisfies Dirichlet boundary conditions $\mathrm{d} X^{1}$ is exact and we can compute

$$
-\frac{i}{2 \pi \alpha^{\prime}} \int_{\Sigma} \check{B}^{\text {res. }}=-\frac{i}{2 \pi \alpha^{\prime}} \int_{\partial \Sigma} 2 \pi \alpha^{\prime}\left[\frac{X^{1}}{2 \pi} \mathrm{d} \check{\chi}_{1}\right]
$$

We can therefore identify a constant Wilson line along the direction $\check{\chi}_{1}$ with the position of the D-brane along the original direction $X^{1}$

$$
\check{\mathrm{a}}=\frac{\left.X^{1}\right|_{\partial \Sigma}}{2 \pi} \mathrm{d} \check{\chi}_{1} .
$$

In summary, the T-dual background is a twisted three-torus with a D1-brane and a constant Wilson line corresponding to the position of D0-brane along the original direction $X^{1}$. 
D1-brane along $X^{2}$. As a second example for a T-duality transformation along a Dirichlet direction we consider a D1-brane along the $X^{2}$-direction. We choose a constant Wilson line for the D1-brane, and the boundary conditions read

$$
0=\left(\mathrm{d} X^{1}\right)_{\tan }, \quad 0=\left(\mathrm{d} X^{2}\right)_{\text {norm }}, \quad 0=\left(\mathrm{d} X^{3}\right)_{\tan }
$$

The constraints (2.8) are solved for instance by

$$
\begin{array}{ll}
a & =a_{2} \mathrm{~d} X^{2}, \\
v_{1} & =0, \\
\omega_{1} & =0,
\end{array} \quad a_{2}=\text { const. }
$$

and the dual background is given again by

$$
\check{\mathrm{G}}_{I J}=\left(\begin{array}{ccc}
\frac{\alpha^{\prime 2}}{R_{1}^{2}} & -\frac{\alpha^{\prime 2}}{R_{1}^{2}} \frac{h}{2 \pi} X^{3} & 0 \\
-\frac{\alpha^{\prime 2}}{R_{1}^{2}} \frac{h}{2 \pi} X^{3} & R_{2}^{2}+\frac{\alpha^{\prime 2}}{R_{1}^{2}}\left[\frac{h}{2 \pi} X^{3}\right]^{2} & 0 \\
0 & 0 & R_{3}^{2}
\end{array}\right), \quad \check{\mathrm{B}}_{I J}=0
$$

with dual basis $\left\{\mathrm{d} \check{\chi}_{1}, \mathrm{~d} X^{2}, \mathrm{~d} X^{3}\right\}$. The boundary conditions (3.32) are evaluated as

$$
\begin{aligned}
& 0=\check{\mathrm{G}}^{11}\left(\mathrm{~d} \check{\chi}_{1}\right)_{\text {norm }}+\check{\mathrm{G}}_{2}{ }_{2}\left(\mathrm{~d} X^{2}\right)_{\text {norm }}, \\
& 0=\check{\mathrm{G}}_{2}{ }^{1}\left(\mathrm{~d} \check{\chi}_{1}\right)_{\text {norm }}+\check{\mathrm{G}}_{22}\left(\mathrm{~d} X^{2}\right)_{\text {norm }}, \\
& 0=\left(\mathrm{d} X^{3}\right)_{\tan },
\end{aligned}
$$

which describe a D2-brane along the directions $\check{\chi}_{1}$ and $X^{2}$. For the residual $B$-field a computation similar to (4.21) applies, which leads to the following dual open-string gauge field

$$
\check{\mathrm{a}}=\frac{\left.X^{1}\right|_{\partial \Sigma}}{2 \pi} \mathrm{d} \check{\chi}_{1}+a_{2} \mathrm{~d} X^{2} .
$$

D2-brane along $\boldsymbol{X}^{\mathbf{2}}-\boldsymbol{X}^{\mathbf{3}}$. For completeness, let us also consider a D2-brane along the directions $X^{2}$ and $X^{3}$ with vanishing open-string field strength. The analysis is very similar to the case of a D1-brane along $X^{2}$ which we just discussed. The dual background is given by (4.25) with dual basis $\left\{\mathrm{d} \check{\chi}_{1}, \mathrm{~d} X^{2}, \mathrm{~d} X^{3}\right\}$. The boundary conditions (3.32) are evaluated as

$$
\begin{aligned}
& 0=\check{\mathrm{G}}^{11}\left(\mathrm{~d} \check{\chi}_{1}\right)_{\text {norm }}+\check{\mathrm{G}}_{2}{ }_{2}\left(\mathrm{~d} X^{2}\right)_{\text {norm }}, \\
& 0=\check{\mathrm{G}}_{2}{ }^{1}\left(\mathrm{~d} \check{\chi}_{1}\right)_{\text {norm }}+\check{\mathrm{G}}_{22}\left(\mathrm{~d} X^{2}\right)_{\text {norm }}, \\
& 0=\left(\mathrm{d} X^{3}\right)_{\text {norm }},
\end{aligned}
$$

which describe a D3-brane along the twisted three-torus. Note that since the $H$-flux of this background vanishes, the Freed-Witten anomaly cancellation condition is satisfied. 


\subsection{Two T-dualities}

In this section we consider two collective T-dualities for the three-torus with $H$-flux defined in (4.1). For concreteness we always perform a collective duality transformation along the directions $X^{1}$ and $X^{2}$, which have the same boundary conditions. However, other combinations can be studied in a similar way.

D2-brane along $\boldsymbol{X}^{\mathbf{1}}-\boldsymbol{X}^{\mathbf{2}}$. We start with a D2-brane along the directions $X^{1}$ and $X^{2}$ with a non-trivial open string field strength $F_{12}=f=$ const. The boundary conditions (2.4) then take the same form as in (4.9), namely

$$
\begin{aligned}
& 0=R_{1}^{2}\left(\mathrm{~d} X^{1}\right)_{\text {norm }}+2 \pi \alpha^{\prime} i\left(f+\frac{h}{4 \pi^{2}} X^{3}\right)\left(\mathrm{d} X^{2}\right)_{\tan }, \\
& 0=R_{2}^{2}\left(\mathrm{~d} X^{2}\right)_{\text {norm }}-2 \pi \alpha^{\prime} i\left(f+\frac{h}{4 \pi^{2}} X^{3}\right)\left(\mathrm{d} X^{1}\right)_{\tan }, \\
& 0=\left(\mathrm{d} X^{3}\right)_{\tan } .
\end{aligned}
$$

For a collective T-duality transformation along two directions the constraints (2.8) and (2.14) are solved by

$$
\begin{aligned}
& a=a_{1} \mathrm{~d} X^{1}+a_{2} \mathrm{~d} X^{2}+\frac{1}{2} f\left(X^{1} \mathrm{~d} X^{2}-X^{2} \mathrm{~d} X^{1}\right), \\
& v_{1}=-2 \pi \alpha^{\prime} f \mathrm{~d} X^{2}, \\
& v_{2}=+2 \pi \alpha^{\prime} f \mathrm{~d} X^{1}, \\
& \omega_{1}=-\pi \alpha^{\prime} f X^{2}, \\
& \omega_{2}=+\pi \alpha^{\prime} f X^{1},
\end{aligned}
$$

and the T-dual metric and $B$-field are determined from the general expressions (3.9) and (3.11) together with our convention (4.3). We find

$$
\begin{aligned}
& \check{\mathrm{G}}_{I J}=\left(\begin{array}{ccc}
\frac{\alpha^{\prime 2} R_{2}^{2}}{R_{1}^{2} R_{2}^{2}+\left[2 \pi \alpha^{\prime} f+\frac{\alpha^{\prime}}{2 \pi} h X^{3}\right]^{2}} & 0 & 0 \\
0 & \frac{\alpha^{\prime 2} R_{1}^{2}}{R_{1}^{2} R_{2}^{2}+\left[2 \pi \alpha^{\prime} f+\frac{\alpha^{\prime}}{2 \pi} h X^{3}\right]^{2}} & 0 \\
0 & R_{3}^{2}
\end{array}\right), \\
& \check{\mathrm{B}}_{I J}=\left(\begin{array}{ccc}
0 & \frac{-\alpha^{\prime 2}\left[2 \pi \alpha^{\prime} f+\frac{\alpha^{\prime}}{2 \pi} h X^{3}\right]}{R_{1}^{2} R_{2}^{2}+\left[2 \pi \alpha^{\prime} f+\frac{\alpha^{\prime}}{2 \pi} h X^{3}\right]^{2}} & 0 \\
\frac{+\alpha^{\prime 2}\left[2 \pi \alpha^{\prime} f+\frac{\alpha^{\prime}}{2 \pi} h X^{3}\right]}{R_{1}^{2} R_{2}^{2}+\left[2 \pi \alpha^{\prime} f+\frac{\alpha^{\prime}}{2 \pi} h X^{3}\right]^{2}} & 0 & 0 \\
0 & 0 & 0
\end{array}\right),
\end{aligned}
$$

which are the metric and $B$-field of the T-fold background [2]. Note that here again the gauge-invariant open-string field strength $2 \pi \alpha^{\prime} \mathcal{F}_{12}=2 \pi \alpha^{\prime} f+\frac{\alpha^{\prime}}{2 \pi} h X^{3}$ appears, and hence the open-string sector has an effect on the T-dual closed-string background. The dual basis is determined via (3.7) and reads $\left\{\mathrm{d} \check{\chi}_{1}, \mathrm{~d} \check{\chi}_{2}, \mathrm{~d} X^{3}\right\}$, and from (3.22) we obtain the boundary conditions

$$
0=\left(\mathrm{d} \check{\chi}_{1}\right)_{\tan }, \quad 0=\left(\mathrm{d} \check{\chi}_{2}\right)_{\tan }, \quad 0=\left(\mathrm{d} X^{3}\right)_{\tan },
$$


describing a D0-brane. The residual $B$-field (3.12) is found as $\check{B}^{\text {res. }}=\mathrm{d} X^{1} \wedge \mathrm{d} \chi_{1}+\mathrm{d} X^{2} \wedge$ $\mathrm{d} \chi_{2}-2 \pi \alpha^{\prime} f \mathrm{~d} X^{1} \wedge \mathrm{d} X^{2}$, which via the computation below (3.26) leads to the condition (3.31)

$$
\left[\check{\chi}_{\alpha}-2 \pi a_{\alpha}\right]_{\partial \Sigma}=0 .
$$

D3-brane along $\boldsymbol{X}^{\mathbf{1}}-\boldsymbol{X}^{\mathbf{2}}-\boldsymbol{X}^{\mathbf{3}}$. For later purposes let us also consider a D3-brane along the three-torus. Since here the Freed-Witten anomaly is not cancelled, this configuration is inconsistent. Nevertheless, applying a collective T-duality transformation along the directions $X^{1}$ and $X^{2}$ gives the T-fold background (4.31) with a D1-brane satisfying the boundary conditions

$$
0=\left(\mathrm{d} \check{\chi}_{1}\right)_{\tan }, \quad 0=\left(\mathrm{d} \check{\chi}_{2}\right)_{\tan }, \quad 0=\left(\mathrm{d} X^{3}\right)_{\text {norm }} .
$$

Since the original configuration is not allowed by the Freed-Witten anomaly, this T-dual configuration is forbidden as well. We come back to this point in section 5.1.

D0-brane. We now turn to collective T-duality transformations along Dirichlet directions. For a D0-brane the boundary conditions take the form

$$
0=\left(\mathrm{d} X^{1}\right)_{\tan }, \quad 0=\left(\mathrm{d} X^{2}\right)_{\tan }, \quad 0=\left(\mathrm{d} X^{3}\right)_{\tan },
$$

and the constraints (2.8) are solved by

$$
a=0, \quad v_{1,2}=0, \quad \omega_{1,2}=0 .
$$

The dual background is then determined by the general expressions (3.9) and (3.11) as

$$
\begin{aligned}
& \check{\mathrm{G}}_{I J}=\left(\begin{array}{ccc}
\frac{\alpha^{\prime 2} R_{2}^{2}}{R_{1}^{2} R_{2}^{2}+\left[\frac{\alpha^{\prime}}{2 \pi} h X^{3}\right]^{2}} & 0 & 0 \\
0 & \frac{\alpha^{\prime 2} R_{1}^{2}}{R_{1}^{2} R_{2}^{2}+\left[\frac{\alpha^{\prime}}{2 \pi} h X^{3}\right]^{2}} & 0 \\
0 & 0 & R_{3}^{2}
\end{array}\right), \\
& \check{\mathrm{B}}_{I J}=\left(\begin{array}{ccc}
0 & \frac{-\alpha^{\prime 2} \frac{\alpha^{\prime}}{2 \pi} h X^{3}}{R_{1}^{2} R_{2}^{2}+\left[\frac{\alpha^{\prime}}{2 \pi} h X^{3}\right]^{2}} & 0 \\
\frac{+\alpha^{\prime 2} \frac{\alpha^{\prime}}{2 \pi} h X^{3}}{R_{1}^{2} R_{2}^{2}+\left[\frac{\alpha^{\prime}}{2 \pi} h X^{3}\right]^{2}} & 0 & 0 \\
0 & 0 & 0
\end{array}\right),
\end{aligned}
$$

which is again that of a T-fold. Note that here the open-string gauge flux $F_{12}=f$ is absent, since the original D0-brane does not support an open-string gauge field. The boundary conditions (3.32) lead to the following expressions

$$
\begin{aligned}
& 0=\check{\mathrm{G}}^{11}\left(\mathrm{~d} \check{\chi}_{1}\right)_{\text {norm }}+i \check{\mathrm{B}}^{12}\left(\mathrm{~d} \check{\chi}_{2}\right)_{\tan }, \\
& 0=\check{\mathrm{G}}^{22}\left(\mathrm{~d} \check{\chi}_{2}\right)_{\text {norm }}+i \check{\mathrm{B}}^{21}\left(\mathrm{~d} \check{\chi}_{1}\right)_{\tan }, \\
& 0=\left(\mathrm{d} X^{3}\right)_{\tan },
\end{aligned}
$$

which take the expected form of Neumann boundary conditions (2.4) for the dual coordinates. 
D1-brane along $\boldsymbol{X}^{\mathbf{3}}$. For completeness we also consider a D1-brane along the $X^{3}$ direction. This configuration is very similar to the case of a D0-brane which we just discussed, and a collective T-duality along the directions $X^{1}$ and $X^{2}$ gives the T-fold background (4.37). The boundary conditions of the dual background describe a D3-brane and are given by

$$
\begin{aligned}
& 0=\check{\mathrm{G}}^{11}\left(\mathrm{~d} \check{\chi}_{1}\right)_{\text {norm }}+i \check{\mathrm{B}}^{12}\left(\mathrm{~d} \check{\chi}_{2}\right)_{\tan }, \\
& 0=\check{\mathrm{G}}^{22}\left(\mathrm{~d} \check{\chi}_{2}\right)_{\text {norm }}+i \check{\mathrm{B}}^{21}\left(\mathrm{~d} \check{\chi}_{1}\right)_{\tan }, \\
& 0=\left(\mathrm{d} X^{3}\right)_{\text {norm }} .
\end{aligned}
$$

\subsection{Three T-dualities}

We finally discuss a collective T-duality transformations for the three-torus along the directions $X^{1}, X^{2}$ and $X^{3}$. As we can see from the second relation in (2.14), in this case the $H$-flux has to vanish and we therefore set $h=0$ in (4.1).

D3-brane along $\boldsymbol{X}^{\mathbf{1}}-\boldsymbol{X}^{\mathbf{2}}-\boldsymbol{X}^{\mathbf{3}}$. Let us start with a D3-brane along all directions of the three-torus, and consider an open-string field strength $F_{12}=f=$ const. together with $B=0$. The boundary conditions (2.4) then read

$$
\begin{aligned}
& 0=R_{1}^{2}\left(\mathrm{~d} X^{1}\right)_{\text {norm }}+2 \pi \alpha^{\prime} i f\left(\mathrm{~d} X^{2}\right)_{\tan }, \\
& 0=R_{2}^{2}\left(\mathrm{~d} X^{2}\right)_{\text {norm }}-2 \pi \alpha^{\prime} i f\left(\mathrm{~d} X^{1}\right)_{\tan }, \\
& 0=R_{3}^{2}\left(\mathrm{~d} X^{3}\right)_{\text {norm }},
\end{aligned}
$$

and the constraints (2.8) and (2.14) are solved by

$$
\begin{aligned}
& a=a_{1} \mathrm{~d} X^{1}+a_{2} \mathrm{~d} X^{2}+a_{3} \mathrm{~d} X^{3}+\frac{1}{2} f\left(X^{1} \mathrm{~d} X^{2}-X^{2} \mathrm{~d} X^{1}\right), \\
& v_{1}=-2 \pi \alpha^{\prime} f \mathrm{~d} X^{2}, \\
& v_{2}=+2 \pi \alpha^{\prime} f \mathrm{~d} X^{1}, \\
& v_{3}=0, \\
& \omega_{1}=-\pi \alpha^{\prime} f X^{2}, a_{2}, a_{3}, f=\text { const. } \\
& \omega_{2}=+\pi \alpha^{\prime} f X^{1}, \\
& \omega_{3}=0 .
\end{aligned}
$$

The T-dual metric and $B$-field are determined from the general expressions (3.9) and (3.11) for which we find

$$
\begin{aligned}
\check{\mathrm{G}}_{I J} & =\left(\begin{array}{ccc}
\frac{\alpha^{\prime 2} R_{2}^{2}}{R_{1}^{2} R_{2}^{2}+\left[2 \pi \alpha^{\prime} f\right]^{2}} & 0 & 0 \\
0 & \frac{\alpha^{\prime 2} R_{1}^{2}}{R_{1}^{2} R_{2}^{2}+\left[2 \pi \alpha^{\prime} f\right]^{2}} & 0 \\
0 & 0 & \frac{\alpha^{\prime 2}}{R_{3}^{2}}
\end{array}\right), \\
\check{\mathrm{B}}_{I J} & =\left(\begin{array}{ccc}
0 & \frac{-2 \pi \alpha^{\prime 3} f}{R_{1}^{2} R_{2}^{2}+\left[2 \pi \alpha^{\prime} f\right]^{2}} & 0 \\
\frac{+2 \pi \alpha^{\prime 3} f}{R_{1}^{2} R_{2}^{2}+\left[2 \pi \alpha^{\prime} f\right]^{2}} & 0 & 0 \\
0 & 0 & 0
\end{array}\right),
\end{aligned}
$$


and we see again that the open-string gauge flux $f$ enters the T-dual closed-string background. The dual basis $\left\{\mathrm{d} \check{\chi}_{1}, \mathrm{~d} \check{\chi}_{2}, \mathrm{~d} \check{\chi}_{3}\right\}$ is subject to the boundary conditions describing a D0-brane

$$
0=\left(\mathrm{d} \check{\chi}_{1}\right)_{\tan }, \quad 0=\left(\mathrm{d} \check{\chi}_{2}\right)_{\tan }, \quad 0=\left(\mathrm{d} \check{\chi}_{3}\right)_{\tan },
$$

where the location of each dual coordinate on the boundary is given by the open-string gauge field as already shown in (4.33).

D0-brane. Finally, for a D0-brane there is no open-string field strength and hence the dual metric and $B$-field after a collective T-duality transformation along all three directions read

$$
\check{\mathrm{G}}_{I J}=\left(\begin{array}{ccc}
\frac{\alpha^{\prime 2}}{R_{1}^{2}} & 0 & 0 \\
0 & \frac{\alpha^{\prime 2}}{R_{2}^{2}} & 0 \\
0 & 0 & \frac{\alpha^{\prime 2}}{R_{3}^{2}}
\end{array}\right), \quad \quad \check{\mathrm{B}}_{I J}=0 .
$$

The dual coordinates are subject to the boundary conditions

$$
0=\left(\mathrm{d} \check{\chi}_{1}\right)_{\text {norm }}, \quad 0=\left(\mathrm{d} \check{\chi}_{2}\right)_{\text {norm }}, \quad 0=\left(\mathrm{d} \check{\chi}_{3}\right)_{\text {norm }},
$$

which describe a D3-brane. The open-string gauge field is characterized by the position of the original D0-brane as in (3.36).

\section{Freed-Witten anomaly and boundary conditions}

We now discuss the results obtained in section 4 . We first briefly review the Freed-Witten anomaly cancellation condition, and then study the global properties of the open-string boundary conditions.

\section{$5.1 \quad$ Freed-Witten anomaly}

It is known that D-branes in backgrounds with non-vanishing $H$-flux are subject to the Freed-Witten anomaly cancellation condition [61]. In particular, the restriction of the field-strength $H=\mathrm{d} B$ to the D-brane has to vanish (in cohomology). Denoting the cycle wrapped by the D-brane by $\Gamma$ and its Poincaré dual by $[\Gamma]$, this condition can be expressed as

$$
H \wedge[\Gamma]=0
$$

For backgrounds with geometric $F$-flux and non-geometric $Q$ - and $R$-fluxes the generalization of this condition has been discussed for instance in [25, 31, 62-65]. Here one finds the expression

$$
(\mathrm{d}-H \wedge-F \circ-Q \bullet-R\llcorner)[\Gamma]=0,
$$


where the various fluxes are interpreted as operators acting in $[\Gamma]$. Using the contraction with a vector field $\iota_{i} \equiv \iota_{\partial_{i}}$, in a coordinate basis they act as

$$
\begin{aligned}
& H \wedge=\frac{1}{3 !} H_{i j k} \mathrm{~d} X^{i} \wedge \mathrm{d} X^{j} \wedge \mathrm{d} X^{k}, \\
& F \circ=\frac{1}{2 !} F^{k}{ }_{i j} \mathrm{~d} X^{i} \wedge \mathrm{d} X^{j} \wedge \iota_{k}, \\
& Q \bullet=\frac{1}{2 !} Q_{i}{ }^{j k} \mathrm{~d} X^{i} \wedge \iota_{j} \wedge \iota_{k}, \\
& R_{\llcorner}=\frac{1}{3 !} R^{i j k} \quad \iota_{i} \wedge \iota_{j} \wedge \iota_{k} .
\end{aligned}
$$

Let us now discuss this condition for the examples studied in section 4 :

- For the three-torus with $H$-flux we mentioned already on page 22 that a D3-brane is forbidden by the Freed-Witten anomaly. And indeed, since $\left[\Gamma_{\mathrm{D} 3}\right]$ is a point on $\mathbb{T}^{3}$ we see that in this case $H \stackrel{!}{=} 0$.

- For the twisted torus we can determine the geometric flux $F^{k}{ }_{i j}$ as the structure constants of the vielbein one-forms under the exterior derivative. We see that for the examples in section 4.1 only $F^{1}{ }_{23}$ is non-vanishing, and hence (5.2) implies that on a twisted $\mathbb{T}^{3}$ a D2-brane along the directions $X^{2}$ and $X^{3}$ is forbidden. This is in agreement with our conclusion on page 22 .

- For the T-fold backgrounds obtained in section 4.2 we can determine the nongeometric $Q$-flux via (3.17). Here we find that the only non-vanishing component is $Q_{3}{ }^{12}$, and hence (5.2) implies that on a T-fold a D1-brane along the $X^{3}$-direction is not allowed. This is again in agreement with our findings on page 26.

\subsection{Boundary conditions}

Next, we consider the global behavior of the open-string boundary conditions for the backgrounds studied in the last section. To do so we first briefly recall how the examples of section 4 can be interpreted as torus fibrations over a circle, and then turn to the global properties of the boundary conditions.

Torus fibrations. We note that the three-torus with $H$-flux, the twisted three-torus and the T-fold background can all be realized as $\mathbb{T}^{2}$-fibrations over a circle. In particular, for the examples studied in section 4 we can express the metric and $B$-field as

$$
G_{i j}=\left(\begin{array}{cc}
G_{\mathrm{ij}}\left(X^{3}\right) & 0 \\
0 & R_{3}^{2}
\end{array}\right), \quad \quad B_{i j}=\left(\begin{array}{cc}
B_{\mathrm{ij}}\left(X^{3}\right) & 0 \\
0 & 0
\end{array}\right),
$$

with $i, j=1,2,3$ and $\mathrm{i}, \mathrm{j}=1,2$ labelling the fiber directions. These fibrations are globally well-defined through gluing local charts with $O(D, D ; \mathbb{Z})$ transformations, which include gauge transformations, diffeomorphisms and so-called $\beta$-transformations. This can be made precise by defining a generalized metric $\mathcal{H}$ which contains the metric and $B$-field as

$$
\mathcal{H}=\left(\begin{array}{cc}
\frac{1}{\alpha^{\prime}}\left(G-B G^{-1} B\right) & +B G^{-1} \\
-G^{-1} B & \alpha^{\prime} G^{-1}
\end{array}\right),
$$


for which we can explicitly check that he examples of section 4 satisfy

$$
\mathcal{H}\left(X^{3}+2 \pi\right)=\mathcal{O}^{-T} \mathcal{H}\left(X^{3}\right) \mathcal{O}^{-1}
$$

Here, $\mathcal{O} \in O(2,2 ; \mathbb{Z}) \subset O(3,3 ; \mathbb{Z})$ are transformations which take the form

$$
\begin{array}{lll}
\mathbb{T}^{3} \text { with } H \text {-flux: } & \mathcal{O}_{\mathrm{B}}=\left(\begin{array}{cc}
\mathbb{1} & 0 \\
\mathrm{~B} & \mathbb{1}
\end{array}\right), & \mathrm{B}=\left(\begin{array}{ccc}
0 & +h & 0 \\
-h & 0 & 0 \\
0 & 0 & 0
\end{array}\right), \\
& \mathcal{O}_{\mathrm{A}}=\left(\begin{array}{cc}
\mathrm{A}^{-1} & 0 \\
0 & \mathrm{~A}^{T}
\end{array}\right), & \mathrm{A}=\left(\begin{array}{ccc}
1 & -h & 0 \\
0 & 1 & 0 \\
0 & 0 & 1
\end{array}\right), \\
\text { Twisted } \mathbb{T}^{3}: & \mathcal{O}_{\beta}=\left(\begin{array}{cc}
\mathbb{1} & \beta \\
0 & \mathbb{1}
\end{array}\right), & \beta=\left(\begin{array}{ccc}
0 & +h & 0 \\
-h & 0 & 0 \\
0 & 0 & 0
\end{array}\right),
\end{array}
$$

and which correspond to gauge transformations, diffeomorphisms and $\beta$-transformations, respectively.

Boundary conditions. Let us now turn to the boundary conditions. Using $2 D$-dimensional matrix notation we can express the Dirichlet and Neumann conditions shown in (2.4) in the following way

$$
\left(\begin{array}{l}
\mathrm{D} \\
\mathrm{N}
\end{array}\right)=\left(\begin{array}{cc}
\alpha^{\prime} & 0 \\
2 \pi \alpha^{\prime} \mathcal{F} & G
\end{array}\right)\left(\begin{array}{c}
i(\mathrm{~d} X)_{\tan } \\
(\mathrm{d} X)_{\mathrm{norm}}
\end{array}\right)
$$

where the restriction of $G$ and $\mathcal{F}$ to the boundary $\partial \Sigma$ is understood. The dilaton can be studied separately and we come back to it below. A particular D-brane configuration is then specified by a projection operator $\Pi$ acting on (5.8), which takes the general form

$$
\Pi=\left(\begin{array}{cc}
\Delta & 0 \\
0 & \mathbb{1}-\Delta
\end{array}\right), \quad \Delta^{2}=\Delta .
$$

For instance, a D1-brane along the $X^{1}$-direction is characterized by the $D \times D$ matrix $\Delta=\operatorname{diag}(0,1, \ldots, 1)$.

We now want to determine how the boundary conditions (5.8) of the three-torus with $H$-flux, twisted three-torus and the T-fold background behave under $X^{3} \rightarrow X^{3}+2 \pi$. For the coordinates we find that under $O(D, D ; \mathbb{Z})$ transformations we have the following general behavior fiber-wise ${ }^{4}$

$$
\left(\begin{array}{c}
i(\mathrm{~d} X)_{\tan } \\
(\mathrm{d} X)_{\text {norm }}
\end{array}\right) \stackrel{\mathcal{O}}{\longrightarrow}\left(\begin{array}{c}
i(\mathrm{~d} \tilde{X})_{\tan } \\
(\mathrm{d} \tilde{X})_{\text {norm }}
\end{array}\right)=\Omega\left(\begin{array}{c}
i(\mathrm{~d} X)_{\tan } \\
(\mathrm{d} X)_{\text {norm }}
\end{array}\right),
$$

\footnotetext{
${ }^{4}$ For a general transformation of the form $\mathcal{O}=\left(\begin{array}{ll}a & b \\ c & d\end{array}\right) \in O(D, D ; \mathbb{Z})$ the matrix $\Omega$ can be determined as $\Omega=\left(\begin{array}{cc}a+2 \pi b \mathcal{F} & \frac{1}{\alpha^{\prime}} b G \\ \frac{1}{\alpha^{\prime}} b G & a+2 \pi b \mathcal{F}\end{array}\right)$, with $G$ the metric and $\mathcal{F}$ the gauge-invariant field-strength.
} 
where the $2 D \times 2 D$ matrix $\Omega$ for each of the cases takes the form

$$
\begin{array}{ll}
\mathbb{T}^{3} \text { with } H \text {-flux: } & \Omega_{\mathrm{B}}=\left(\begin{array}{cc}
\mathbb{1} & 0 \\
0 & \mathbb{1}
\end{array}\right), \\
& \\
\text { twisted } \mathbb{T}^{3}: & \Omega_{\mathrm{A}}=\left(\begin{array}{cc}
\mathrm{A}^{-1} & 0 \\
0 & \mathrm{~A}^{-1}
\end{array}\right), \\
\text { T-fold: } & \Omega_{\beta}=\left(\begin{array}{cc}
\mathbb{1}+2 \pi \beta \mathcal{F} & \frac{1}{\alpha^{\prime}} \beta G \\
\frac{1}{\alpha^{\prime}} \beta G & \mathbb{1}+2 \pi \beta \mathcal{F}
\end{array}\right) .
\end{array}
$$

The matrices $\mathrm{A}$ and $\beta$ have been defined in (5.7), and we note that for the case of the T-fold the normal and tangential part of $\mathrm{d} X^{i}$ are mixed under the $O(D, D ; \mathbb{Z})$ transformation. Using these relations, for the examples of section 4 we then find

$$
\begin{aligned}
\left(\begin{array}{c}
\mathrm{D} \\
\mathrm{N}
\end{array}\right)_{X^{3}+2 \pi} & =\left(\begin{array}{cc}
\alpha^{\prime} & 0 \\
2 \pi \alpha^{\prime} \mathcal{F} & G
\end{array}\right)_{X^{3}+2 \pi}\left(\begin{array}{c}
i(\mathrm{~d} \tilde{X})_{\tan } \\
(\mathrm{d} \tilde{X})_{\text {norm }}
\end{array}\right) \\
& =\mathcal{O}_{\star}\left(\begin{array}{cc}
\alpha^{\prime} & 0 \\
2 \pi \alpha^{\prime} \mathcal{F} & G
\end{array}\right)_{X^{3}} \Omega_{\star}^{-1}\left(\begin{array}{c}
i(\mathrm{~d} \tilde{X})_{\tan } \\
(\mathrm{d} \tilde{X})_{\text {norm }}
\end{array}\right) \\
& =\mathcal{O}_{\star}\left(\begin{array}{c}
\mathrm{D} \\
\mathrm{N}
\end{array}\right)_{X^{3}},
\end{aligned}
$$

where the subscript $\star=(\mathrm{B}, \mathrm{A}, \beta)$ corresponds to the three-torus with $H$-flux, the twisted $\mathbb{T}^{3}$ and the T-fold. The coordinates $\mathrm{d} \tilde{X}^{i}$ in one patch are related to $\mathrm{d} X^{i}$ in another patch via (5.10), and we emphasize that these relations are to be evaluated on the boundary. We then see that the boundary conditions are globally-well defined using, respectively, gauge transformations, diffeomorphisms and $\beta$-transformations.

Dilaton. In the expression (5.8) for the open-string boundary conditions we have omitted the dilaton. This contribution can be discussed separately, and we first determine using (3.13)

$$
\begin{array}{ll}
\mathbb{T}^{3} \text { with } H \text {-flux: } & \phi=\phi_{0}, \\
\text { twisted } \mathbb{T}^{3}: & \phi=\phi_{0}-\log \left[\frac{R_{1}}{\sqrt{\alpha^{\prime}}}\right], \\
\text { T-fold: } & \phi=\phi_{0}-\frac{1}{2} \log \left[\frac{R_{1}^{2} R_{2}^{2}}{\alpha^{2}}+\left(2 \pi f+\frac{h}{2 \pi} X^{3}\right)^{2}\right],
\end{array}
$$

where for the T-fold we included the open-string field strength $f=$ const. which in some examples vanishes. We now consider each of these cases separately:

- For the three torus with $H$-flux, a gauge transformation leaves the metric invariant, and hence the combination $e^{-2 \phi} \sqrt{\operatorname{det} G}$ is invariant under the action of $\mathcal{O}_{\mathrm{B}}$. Further- 
more, since the dilaton is constant it does not change under $X^{3} \rightarrow X^{3}+2 \pi$ and so the contribution to the corresponding boundary conditions is well-defined.

- For the twisted three-torus $e^{-2 \phi} \sqrt{\operatorname{det} G}$ is invariant under diffeomorphisms $\mathcal{O}_{\mathrm{A}}$ and the dilaton is constant, so the contribution to the boundary conditions is again welldefined.

- For the T-fold on the other hand, the dilaton is not constant and transforms under $\beta$-transformations. In particular, by requiring $e^{-2 \phi} \sqrt{\operatorname{det} G}$ to be invariant we can determine

$$
\phi\left(X^{3}+2 \pi\right)=\mathcal{O}_{\beta}\left[\phi\left(X^{3}\right)\right]
$$

where the action of $\mathcal{O}_{\beta}$ is understood in an abstract way and not as a matrix multiplication. We therefore see that the dilaton is well-defined under $X^{3} \rightarrow X^{3}+2 \pi$ using a $\beta$-transformation, and hence also the contribution to the boundary conditions is well-defined.

Projection. So far we have studied how (5.8) behaves under $X^{3} \rightarrow X^{3}+2 \pi$ for the examples of section 4. We now want to discuss how the projection operator (5.9) is implemented on the boundary conditions. To do so, we again proceed by discussing the examples:

- For the three-torus with $H$-flux the behavior under $X^{3} \rightarrow X^{3}+2 \pi$ is captured by $(5.12)$, provided that first the $\mathcal{O}_{\mathrm{B}} \in O(3,3 ; \mathbb{Z})$ transformation is performed and after that the projection (5.9). In particular, we have

$$
\Pi\left[\left(\begin{array}{l}
\mathrm{D} \\
\mathrm{N}
\end{array}\right)_{X^{3}+2 \pi}\right]=\Pi\left[\mathcal{O}_{\mathrm{B}}\left(\begin{array}{l}
\mathrm{D} \\
\mathrm{N}
\end{array}\right)_{X^{3}}\right] .
$$

One quickly sees for instance from the $(\mathrm{NN})$ case that if we perform the $\mathcal{O}_{\mathrm{B}}$ transformation on the projected boundary conditions we do not reproduce the expected result from (5.12).

- For the twisted three-torus a similar analysis can be made. We verified explicitly that a projection similar to (5.15) produces the expected behavior of the boundary conditions from (5.12), and that performing the $\mathcal{O}_{\mathrm{A}}$ transformation on the projected boundary conditions does not match with the explicit computation.

- Finally, for the T-fold the condition (5.15) similarly applies. This means in particular, that the type of D-brane does not change under the identification $X^{3} \rightarrow X^{3}+2 \pi$. The boundary conditions are therefore well-defined.

\section{Summary and conclusion}

In this paper we have studied T-duality transformations for open-string backgrounds via Buscher's procedure. We illustrated this formalism with the example of the three-torus with $H$-flux and its T-dual configurations, and we analyzed global properties of the open-string boundary conditions for these backgrounds. More concretely: 
- T-duality transformations for open strings via Buscher's procedure have been discussed before in the literature $[35,36,38]$. Here we extended these analyses and worked-out missing details: we took into account non-trivial world-sheet topologies, we included T-duality along directions with Dirichlet boundary conditions, and we allowed for collective T-duality transformations along multiple directions.

We find that - as expected - also for curved backgrounds Neumann and Dirichlet boundary conditions are interchanged under T-duality, and that a constant openstring Wilson line along a Neumann direction shifts the position of the D-brane in the T-dual Dirichlet direction and vice versa.

- In section 4 we illustrated the above formalism through various examples for the threetorus with $H$-flux. We obtained D-brane configurations on the twisted three-torus and on the T-fold, and we saw that an open-string gauge-flux affects the closed-string sector of the T-dual theory.

- In section 5 we discussed the results of section 4. After briefly reviewing the FreedWitten anomaly cancellation condition, we showed that D-brane boundary conditions for the three-torus with $H$-flux, the twisted three-torus and for the T-fold are globally well-defined using, respectively, gauge transformations, diffeomorphisms and $\beta$-transformations.

Since $\beta$-transformations mix the tangential and normal part of $\mathrm{d} X^{i}$ on the boundary, naively one might have thought that $\mathrm{D} p$-branes on the T-fold can change their dimensionality under $X^{3} \rightarrow X^{3}+2 \pi$. However, we show that this is not true due to the mixing between the metric and $B$-field under $\beta$-transformations. Our findings furthermore agree with results obtained in doubled geometry in $[2,32,33]$.

An interesting next step is to extend our formalism to non-abelian T-duality transformations [56, 57, 66-69]. We have already included the possibility of a non-abelian isometry algebra for the gauging procedure and for integrating-out the gauge fields, however, the change of basis (3.7) is singular in certain non-abelian cases. One approach to avoid this problem is to find a different change of basis which is non-singular, and we hope to come back to this question in the future.

\section{Acknowledgments}

We would like to thank R. Blumenhagen, S. Krippendorf, C. Mayrhofer and R. Szabo for helpful discussions. The work of F.C.-T. was funded by the CONICYT scholarship 72160340 from the Government of Chile. The work of D.L. is supported by the ERC Advanced Grant "Strings and Gravity" (Grant No. 320045) and the Excellence Cluster Universe. He also is grateful to the CERN theory department for its hospitality, when part of this work was performed.

Open Access. This article is distributed under the terms of the Creative Commons Attribution License (CC-BY 4.0), which permits any use, distribution and reproduction in any medium, provided the original author(s) and source are credited. 


\section{References}

[1] S. Hellerman, J. McGreevy and B. Williams, Geometric constructions of nongeometric string theories, JHEP 01 (2004) 024 [hep-th/0208174] [INSPIRE].

[2] C.M. Hull, A geometry for non-geometric string backgrounds, JHEP 10 (2005) 065 [hep-th/0406102] [INSPIRE].

[3] K. Dasgupta, G. Rajesh and S. Sethi, M theory, orientifolds and G-flux, JHEP 08 (1999) 023 [hep-th/9908088] [INSPIRE].

[4] S. Kachru, M.B. Schulz, P.K. Tripathy and S.P. Trivedi, New supersymmetric string compactifications, JHEP 03 (2003) 061 [hep-th/0211182] [INSPIRE].

[5] J. Shelton, W. Taylor and B. Wecht, Nongeometric flux compactifications, JHEP 10 (2005) 085 [hep-th/0508133] [INSPIRE].

[6] J. Shelton, W. Taylor and B. Wecht, Generalized Flux Vacua, JHEP 02 (2007) 095 [hep-th/0607015] [INSPIRE].

[7] V. Mathai and J.M. Rosenberg, T duality for torus bundles with H fluxes via noncommutative topology, Commun. Math. Phys. 253 (2004) 705 [hep-th/0401168] [INSPIRE].

[8] V. Mathai and J.M. Rosenberg, On mysteriously missing T-duals, H-flux and the T-duality group, in Differential geometry and physics. Proceedings, 23rd International Conference, Tianjin, China, August 20-26, 2005, pp. 350-358, hep-th/0409073 [INSPIRE].

[9] D. Lüst, T-duality and closed string non-commutative (doubled) geometry, JHEP 12 (2010) 084 [arXiv: 1010.1361] [INSPIRE].

[10] C. Condeescu, I. Florakis and D. Lüst, Asymmetric Orbifolds, Non-Geometric Fluxes and Non-Commutativity in Closed String Theory, JHEP 04 (2012) 121 [arXiv:1202.6366] [INSPIRE].

[11] D. Andriot, O. Hohm, M. Larfors, D. Lüst and P. Patalong, Non-Geometric Fluxes in Supergravity and Double Field Theory, Fortsch. Phys. 60 (2012) 1150 [arXiv:1204.1979] [INSPIRE].

[12] D. Andriot, M. Larfors, D. Lüst and P. Patalong, (Non-)commutative closed string on T-dual toroidal backgrounds, JHEP 06 (2013) 021 [arXiv: 1211.6437] [INSPIRE].

[13] C.D.A. Blair, Non-commutativity and non-associativity of the doubled string in non-geometric backgrounds, JHEP 06 (2015) 091 [arXiv:1405.2283] [INSPIRE].

[14] P. Bouwknegt, K. Hannabuss and V. Mathai, Nonassociative tori and applications to T-duality, Commun. Math. Phys. 264 (2006) 41 [hep-th/0412092] [InSPIRE].

[15] I. Ellwood and A. Hashimoto, Effective descriptions of branes on non-geometric tori, JHEP 12 (2006) 025 [hep-th/0607135] [INSPIRE].

[16] R. Blumenhagen and E. Plauschinn, Nonassociative Gravity in String Theory?, J. Phys. A 44 (2011) 015401 [arXiv: 1010.1263] [INSPIRE].

[17] R. Blumenhagen, A. Deser, D. Lüst, E. Plauschinn and F. Rennecke, Non-geometric Fluxes, Asymmetric Strings and Nonassociative Geometry, J. Phys. A 44 (2011) 385401 [arXiv: 1106.0316] [INSPIRE].

[18] E. Plauschinn, Non-geometric fluxes and non-associative geometry, PoS(CORFU2011) 061 [arXiv: 1203.6203] [INSPIRE]. 
[19] D. Mylonas, P. Schupp and R.J. Szabo, Membrane sigma-models and Quantization of Non-Geometric Flux Backgrounds, JHEP 09 (2012) 012 [arXiv:1207.0926] [INSPIRE].

[20] I. Bakas and D. Lüst, 3-Cocycles, Non-Associative Star-Products and the Magnetic Paradigm of R-Flux String Vacua, JHEP 01 (2014) 171 [arXiv: 1309.3172] [INSPIRE].

[21] D. Mylonas, P. Schupp and R.J. Szabo, Non-Geometric Fluxes, Quasi-Hopf Twist Deformations and Nonassociative Quantum Mechanics, J. Math. Phys. 55 (2014) 122301 [arXiv:1312.1621] [INSPIRE].

[22] A. Chatzistavrakidis, L. Jonke and O. Lechtenfeld, Sigma-models for genuinely non-geometric backgrounds, JHEP 11 (2015) 182 [arXiv:1505.05457] [INSPIRE].

[23] R.J. Szabo, Higher Quantum Geometry and Non-Geometric String Theory, in 17th Hellenic School and Workshops on Elementary Particle Physics and Gravity (CORFU2017) Corfu, Greece, September 2-28, 2017, 2018, arXiv:1803.08861 [InSPIRE].

[24] G. Aldazabal, P.G. Camara, A. Font and L.E. Ibáñez, More dual fluxes and moduli fixing, JHEP 05 (2006) 070 [hep-th/0602089] [INSPIRE].

[25] G. Villadoro and F. Zwirner, D terms from D-branes, gauge invariance and moduli stabilization in flux compactifications, JHEP 03 (2006) 087 [hep-th/0602120] [INSPIRE].

[26] A. Micu, E. Palti and G. Tasinato, Towards Minkowski Vacua in Type II String Compactifications, JHEP 03 (2007) 104 [hep-th/0701173] [INSPIRE].

[27] A. Font, A. Guarino and J.M. Moreno, Algebras and non-geometric flux vacua, JHEP 12 (2008) 050 [arXiv: 0809.3748] [INSPIRE].

[28] C. Caviezel, T. Wrase and M. Zagermann, Moduli Stabilization and Cosmology of Type IIB on $\mathrm{SU}(2)$-Structure Orientifolds, JHEP 04 (2010) 011 [arXiv:0912.3287] [INSPIRE].

[29] G. Dibitetto, A. Guarino and D. Roest, Charting the landscape of $N=4$ flux compactifications, JHEP 03 (2011) 137 [arXiv:1102.0239] [INSPIRE].

[30] F. Hassler, D. Lüst and S. Massai, On Inflation and de Sitter in Non-Geometric String Backgrounds, Fortsch. Phys. 65 (2017) 1700062 [arXiv:1405.2325] [INSPIRE].

[31] R. Blumenhagen et al., A Flux-Scaling Scenario for High-Scale Moduli Stabilization in String Theory, Nucl. Phys. B 897 (2015) 500 [arXiv:1503.07634] [INSPIRE].

[32] A. Lawrence, M.B. Schulz and B. Wecht, D-branes in nongeometric backgrounds, JHEP 07 (2006) 038 [hep-th/0602025] [INSPIRE].

[33] C. Albertsson, T. Kimura and R.A. Reid-Edwards, D-branes and doubled geometry, JHEP 04 (2009) 113 [arXiv: 0806.1783] [inSPIRE].

[34] T.H. Buscher, A Symmetry of the String Background Field Equations, Phys. Lett. B 194 (1987) 59 [INSPIRE].

[35] E. Alvarez, J.L.F. Barbon and J. Borlaf, T duality for open strings, Nucl. Phys. B 479 (1996) 218 [hep-th/9603089] [INSPIRE].

[36] H. Dorn and H.J. Otto, On T duality for open strings in general Abelian and nonAbelian gauge field backgrounds, Phys. Lett. B 381 (1996) 81 [hep-th/9603186] [INSPIRE].

[37] H. Dorn and H.J. Otto, Remarks on T duality for open strings, Nucl. Phys. Proc. Suppl. B 56 (1997) 30 [hep-th/9702018] [INSPIRE]. 
[38] S. Förste, A.A. Kehagias and S. Schwager, NonAbelian duality for open strings, Nucl. Phys. B 478 (1996) 141 [hep-th/9604013] [INSPIRE].

[39] S. Förste, A.A. Kehagias and S. Schwager, NonAbelian T duality for open strings, Nucl. Phys. Proc. Suppl. B 56 (1997) 36 [hep-th/9610062] [INSPIRE].

[40] S. Förste, A.A. Kehagias and S. Schwager, $T$ duality for open strings with respect to nonAbelian isometries, in Gauge theories, applied supersymmetry and quantum gravity. Proceedings, 2nd Conference, London, U.K., July 5-10, 1996, pp. 271-278, hep-th/9611060 [INSPIRE].

[41] C. Albertsson, U. Lindström and M. Zabzine, T-duality for the sigma model with boundaries, JHEP 12 (2004) 056 [hep-th/0410217] [INSPIRE].

[42] J. Borlaf and Y. Lozano, Aspects of T duality in open strings, Nucl. Phys. B 480 (1996) 239 [hep-th/9607051] [INSPIRE].

[43] Y. Lozano, Duality and canonical transformations, Mod. Phys. Lett. A 11 (1996) 2893 [hep-th/9610024] [INSPIRE].

[44] A.A. Tseytlin, Selfduality of Born-Infeld action and Dirichlet three-brane of type IIB superstring theory, Nucl. Phys. B 469 (1996) 51 [hep-th/9602064] [INSPIRE].

[45] E. Bergshoeff and M. De Roo, D-branes and T duality, Phys. Lett. B 380 (1996) 265 [hep-th/9603123] [INSPIRE].

[46] M.B. Green, C.M. Hull and P.K. Townsend, D-brane Wess-Zumino actions, $t$ duality and the cosmological constant, Phys. Lett. B 382 (1996) 65 [hep-th/9604119] [INSPIRE].

[47] S. Kawai and Y. Sugawara, D-branes in T-fold conformal field theory, JHEP 02 (2008) 027 [arXiv: 0709.0257] [INSPIRE].

[48] P. Grange and R. Minasian, Tachyon condensation and D-branes in generalized geometries, Nucl. Phys. B 741 (2006) 199 [hep-th/0512185] [INSPIRE].

[49] C. Klimčík and P. Ševera, Poisson Lie T duality: Open strings and D-branes, Phys. Lett. B 376 (1996) 82 [hep-th/9512124] [INSPIRE].

[50] C. Albertsson and R.A. Reid-Edwards, Worldsheet boundary conditions in Poisson-Lie T-duality, JHEP 03 (2007) 004 [hep-th/0606024] [INSPIRE].

[51] L. Davidović, Open string T-duality in a weakly curved background, Eur. Phys. J. C 76 (2016) 660 [arXiv: 1603.06411] [INSPIRE].

[52] B. Sazdović, From geometry to non-geometry via T-duality, Chin. Phys. C 42 (2018) 083106 [arXiv: 1606.01938] [INSPIRE].

[53] B. Sazdović, Open string T-duality in double space, Eur. Phys. J. C 77 (2017) 634 [arXiv:1704.01163] [INSPIRE].

[54] S. Cappell, D. DeTurck, H. Gluck and E.Y. Miller, Cohomology of Harmonic Forms on Riemannian Manifolds With Boundary, math/0508372 [INSPIRE].

[55] M. Roček and E.P. Verlinde, Duality, quotients and currents, Nucl. Phys. B 373 (1992) 630 [hep-th/9110053] [INSPIRE].

[56] A. Giveon and M. Roček, On nonAbelian duality, Nucl. Phys. B 421 (1994) 173 [hep-th/9308154] [INSPIRE]. 
[57] E. Alvarez, L. Álvarez-Gaumé, J.L.F. Barbon and Y. Lozano, Some global aspects of duality in string theory, Nucl. Phys. B 415 (1994) 71 [hep-th/9309039] [INSPIRE].

[58] E. Plauschinn, On T-duality transformations for the three-sphere, Nucl. Phys. B 893 (2015) 257 [arXiv: 1408.1715] [INSPIRE].

[59] T.H. Buscher, Path integral derivation of quantum duality in nonlinear sigma-models, Phys. Lett. B 201 (1988) 466 [INSPIRE].

[60] E. Plauschinn, T-duality revisited, JHEP 01 (2014) 131 [arXiv:1310.4194] [InSPIRE].

[61] D.S. Freed and E. Witten, Anomalies in string theory with D-branes, Asian J. Math. 3 (1999) 819 [hep-th/9907189] [INSPIRE].

[62] P.G. Camara, A. Font and L.E. Ibáñez, Fluxes, moduli fixing and MSSM-like vacua in a simple IIA orientifold, JHEP 09 (2005) 013 [hep-th/0506066] [INSPIRE].

[63] O. Loaiza-Brito, Freed-Witten anomaly in general flux compactification, Phys. Rev. D 76 (2007) 106015 [hep-th/0612088] [INSPIRE].

[64] G. Aldazabal, P.G. Camara and J.A. Rosabal, Flux algebra, Bianchi identities and Freed-Witten anomalies in F-theory compactifications, Nucl. Phys. B 814 (2009) 21 [arXiv:0811.2900] [INSPIRE].

[65] G. Aldazabal, D. Marques, C. Núñez and J.A. Rosabal, On type IIB moduli stabilization and $N=4,8$ supergravities, Nucl. Phys. B 849 (2011) 80 [arXiv:1101.5954] [INSPIRE].

[66] X.C. de la Ossa and F. Quevedo, Duality symmetries from nonAbelian isometries in string theory, Nucl. Phys. B 403 (1993) 377 [hep-th/9210021] [InSPIRE].

[67] K. Sfetsos, Gauged WZW models and nonAbelian duality, Phys. Rev. D 50 (1994) 2784 [hep-th/9402031] [INSPIRE].

[68] E. Alvarez, L. Álvarez-Gaumé and Y. Lozano, On nonAbelian duality, Nucl. Phys. B 424 (1994) 155 [hep-th/9403155] [INSPIRE].

[69] Y. Lozano, NonAbelian duality and canonical transformations, Phys. Lett. B 355 (1995) 165 [hep-th/9503045] [INSPIRE]. 